\title{
Bullying y ciberbullying: victimización, acoso y daño. Necesidad de intervenir en el entorno escolar
}

\section{Bullying and cyberbullying: victimisation, harassment, and harm. The need to intervene in the educational centre}

\begin{abstract}
Dra. Maialen GARMENDIA LARRAÑAGA. Profesora Titular. Universidad del País Vasco (maialen.garmendia@ehu.eus). Dra. Estefanía JIMÉNEZ IGLESIAS. Profesora Agregada. Universidad del País Vasco (estefania.jimenez@ehu.eus). Dra. Nekane LARRAÑAGA AIZPURU. Profesora Agregada. Universidad del País Vasco (nekane.larranaga@ehu.eus).
\end{abstract}

\section{Resumen:}

El presente trabajo analiza el ciberbullying a través de los resultados de una encuesta realizada a una muestra representativa de los y las menores españolas usuarias de Internet de entre 9 y 16 años. Los datos revelan que el acoso a través de las tecnologías digitales forma parte de un clima de violencia entre los preadolescente y adolescentes, entre los cuales el acoso cara a cara prevalece claramente sobre el que tiene lugar a través de medios tecnológicos. Si bien las diversas modalidades de acoso se superponen entre sí, la más frecuente es la que tiene lugar en entornos offline. La incidencia del ciberbullying está fuertemente estructurada por la edad y aumenta en función de esta. Se aprecia un intercambio de roles entre los y las menores implicados en los episodios violentos online, dado que tres de cada cuatro acosadores han sido también víctimas de acoso. Resultan particularmente llamativos los datos del grupo de 13-14 años, en el que la prevalencia de la participación en el ciberbullying es relativamente elevada y además los y las menores reconocen haberse sentido muy disgustados por su victimización. Los resultados avalan la necesidad de intervenir desde el entorno escolar para minimizar la incidencia del acoso, ya que es el principal ámbito en el que tiene lugar, y además este es el ámbito más igualitario y efectivo.

Palabras clave: acoso, adolescentes, ciberacoso, convivencia escolar, Internet, dispositivos móviles, riesgos.

Fecha de recepción de la versión definitiva de este artículo: 03-03-2019.

Cómo citar este artículo: Garmendia Larrañaga, M., Jiménez Iglesias., E. y Larrañaga Aizpuru, N. (2019). Bullying y ciberbullying: victimización, acoso y daño. Necesidad de intervenir en el entorno escolar | Bullying and cyberbullying: victimisation, harassment, and harm. The need to intervene in the educational centre. Revista Española de Pedagogia, 77 (273), 295-312. doi: https://doi.org/10.22550/REP77-2-2019-08 


\section{Abstract:}

This work focusses on cyberbullying, analysing the results of a survey administered to a representative sample of Spanish Internet users aged between 9 and 16 . The data show that harassment on digital devices is part of the climate of violence among pre-teenagers and teenagers, where face-to-face bullying is much higher than online bullying. Although bullying occurs in different ways, and these tend to overlap, the most frequent form is offline bullying. The prevalence of cyberbullying varies considerably by age, tending to increase as the subjects' age increases, whereas offline bullying decreases among 15-16 year olds. The boundary between victims and perpetrators is difficult to sketch in cyberbullying as three out of four children who admit having treated others in a hurtful or nasty way on the internet or with mobile phones have themselves been treated in this way by others. The evidence regarding 13-14 year olds is especially worrying as they are more involved in cyberbullying and a great many of them say they have felt very upset when victimized. The results display a need to prevent and deal with cyberbullying at school, as this is the most effective and equitable site for intervention.

Keywords: bullying, teenagers, cyberbullying, school life, Internet, mobile devices, risks.
1. Introducción y estado de la cuestión

El interés y la preocupación social e institucional por las conductas violentas entre escolares se ha incrementado en los últimos años, y del mismo modo lo ha hecho su presencia en los medios de comunicación (Sahuquillo, 2017). Tanto el bullying cara a cara como el acoso que se desarrolla mediante las TIC son objeto de análisis y preocupación a distintos niveles, lo que ha desembocado en una diversidad de definiciones operativas a la hora de describir ambos fenómenos.

Si bien hay que entender el ciberbullying como una extensión del acoso escolar tradicional que acontece en las redes sociales virtuales e Internet, algunas de las características del bullying - repetición, desequilibrio de poder, intencionalidad y falta de justificación - requieren de una redefinición en el caso del ciberbullying u acoso online. Se señala, por ejemplo, la repetición como criterio discutido, dado que en ocasiones una única agresión a través de medios tecnológicos puede extenderse y perpetuarse en la red, aunque solo haya habido una única actuación por parte del agresor (Levy et al., 2012; Menesini et al., 2012; Slonje, Smith y Frisén, 2013). Igualmente, factores como el anonimato bajo el cual puede ocultarse el acosador (Hinduja y Patchin, 2008), la dificultad de la víctima para dejar de ser acosada a través de Internet (Ovejero, Smith y Yubero, 2013), y la facilidad con la que se amplifica la audiencia en el caso del ciberbullying, contribuyen a que la indefensión de la víctima y su perjuicio sean mayores que en los casos de acoso tradicional (Estévez, Villardón, Calvete, Padilla y Orue, 2010). 
Aunque el acoso puede producirse en escenarios ajenos a la escuela, en espacios públicos como parques o centros deportivos, y el contexto online trasciende los centros escolares, la escuela es el entorno en el que los niños y niñas desarrollan la mayor parte de sus relaciones sociales, con lo que constituye el lugar donde las conductas de acoso, tradicional y online, son más habituales y visibles.

Entre los elementos relacionados con el acoso - el entorno familiar, las características cognitivas de acosadores y víctimas 0 factores sociales- cabe mencionar aquellos vinculados al entorno escolar como el rendimiento académico, la influencia interpares, la falta de seguridad y supervisión en las escuelas, la falta de medidas de atención a la diversidad y la ausencia de un clima positivo en el centro escolar y el desconocimiento y escaso control de las relaciones que el alumnado mantiene en las redes sociales que, según señalan Cook, Williams, Guerra, Kim y Sadek (2010), constituyen factores de riesgo de ciberbullying.

No hay consenso a la hora de cuantificar la prevalencia de bullying y ciberbullying entre los escolares españoles. Los datos pueden variar, y de hecho, varían según cuáles sean las herramientas para medirlos, la definición del fenómeno o la franja de edad considerada, como señala Smith (2016). A nivel mundial la OMS reportaba en 2010 una prevalencia del acoso escolar que fluctuaba en Europa, según países, entre el $13 \%$ y el $27 \%$, con datos similares para 2016 (Currie et al., 2012; Inchley, $\mathrm{Cu}$ rrie, Young, Oddrun y Torbjorn, 2016).
Respecto al ciberacoso, Zych, Ortega-Ruiz y Del Rey (2015) dedujeron de la revisión de más de 60 estudios que al menos uno de cada cinco-siete menores están implicados. A escala europea Livingstone, Haddon, Görzig y Ólafsson (2011) determinaron que por término medio un $6 \%$ de la población de entre 9 y 16 años considera haber sido víctima de ciberacoso, $\mathrm{y}$ el $3 \%$ reconocía haber acosado a través de Internet. En el ámbito español, Garmendia, Jiménez, Casado y Mascheroni (2016) constataron que la prevalencia de la victimización online era del $12 \%$ y la del acoso del $8 \%$ entre los y las menores usuarios de Internet de entre 9 y 16 años. Igualmente, destacamos por la amplitud de su muestra la encuesta desarrollada en 2016 por Save the Children, que incluyó a más de 21000 estudiantes españoles de centros públicos de entre 12 y 16 años que ofreció como resultado que un $9.3 \%$ de los encuestados consideraba que había sufrido acoso tradicional en los dos últimos meses, y un $6.9 \%$ se consideraba víctima de ciberacoso, siendo el insulto la forma de violencia más recurrente (Save the Children, 2016).

Las vías utilizadas para perpetrar una agresión online son diversas: acoso telefónico, grabaciones de vejaciones físicas difundidas a través de mensajería instantánea o plataformas para compartir, correos electrónicos, difusión de rumores y amenazas en redes sociales, exclusión, etc. En este sentido, a medida que los hábitos online de la población escolar y su acceso a diferentes elementos tecnológicos han ido aumentando y diversificándose, también lo han hecho las modalidades de ciberbullying. Cabe destacar que en la actualidad, el 
uso de Internet está extendido a prácticamente toda la población escolar: en España, por término medio, el $95 \%$ de los niños y niñas de 10 a 15 años usa Internet. El uso de los teléfonos móviles, por su parte, está fuertemente estructurado en función de la edad: un $25 \%$ de los niños y niñas disponen de móvil a los 10 años; prácticamente la mitad lo hacen a los 11, y a partir de los 14 años más del $90 \%$ tiene un dispositivo para su propio uso (Instituto Nacional de Estadística, 2017). En Europa, el 97\% de la población de 15 a 24 años tiene acceso prácticamente diario a Internet, y el $85 \%$ lo hace desde su smartphone (European Commission, 2015).

La escuela es el ámbito clave de actuación sobre el acoso entre menores y se reclama la necesidad de su intervención ante este fenómeno. Sin embargo, la intervención escolar sobre el acoso afronta hoy en día retos complejos. Se dan situaciones, por ejemplo, como que más de un centro escolar esté implicado en un mismo caso de ciberacoso o que por considerar que este ha ocurrido fuera de la escuela se diluyen las responsabilidades sin que nadie actúe. Sin embargo, el acoso causa daño a toda una comunidad, luego es necesario que todas las partes, tanto la escuela, como la familia o la sociedad, se impliquen (Cohen-Almagor, 2018). El trabajo de prevención contra distintas formas de violencia entre todo el alumnado resulta fundamental de cara a evitar cualquier forma de acoso (Save the Children, 2016).

Desde la psicología se propone que el papel de los centros escolares constituya un esfuerzo cooperativo entre diferentes partes como la escuela y la familia además de la sociedad o la comunidad. La intervención se ha orientar tanto hacia el agresor como hacia la víctima y sobre ambos en conjunto. Se recomiendan distintos tipos de intervención, tanto de prevención del conflicto como de mejora de la convivencia escolar: intervención primaria, para detectar situaciones iniciales de maltrato, e intervención secundaria ante situaciones consolidadas, aportando apoyo terapéutico o protección a las víctimas y controlando a agresores (Garaigordobil, 2011). Sin embargo, la mejor forma de intervención que se propone es el fomento de la convivencia en las escuelas, estimulando competencias sociales y emocionales, la cooperación y la resolución de conflictos (Garaigordobil, 2015).

Del Rey, Estévez, y Ojeda (2018) hacen referencia a diferentes programas escolares centrados en la prevención 0 intervención ante el ciberbullying. Estos programas están orientados a sensibilizar a la sociedad y a hacer reflexionar al alumnado, reforzando una actitud crítica ante el fenómeno y concienciándoles sobre la seguridad y la protección que han de tener en Internet, potenciando el buen uso de las redes sociales e Internet (Del Rey et al., 2018).

Las evidencias muestran que en grados variables el cyberbullying complementa el acoso escolar (Hinduja y Patchin, 2009; Smith, Kwak y Toda, 2016). El propósito de este trabajo consiste en analizar la relación existente entre bullying y ciberbullying en el contexto español, partiendo de la base de que existe una superposición entre ambos fenómenos, así como describir la frecuencia en la que se producen en di- 
ferentes franjas de edad y con distintos niveles de daño para sus víctimas, y explorar la asociación entre los roles de víctima y acosador en los procesos de acoso, tomando como contexto el entorno escolar.

\subsection{Material y métodos}

Este artículo analiza los datos obtenidos a través de una encuesta financiada por el proyecto del MINECO CSO 201347304-R que se realizó entre abril y junio de 2015. Respecto a la población y muestra, según los datos del INE, se estimaba que la población de referencia - menores de entre 9 y 16 años—era de 3758 400. Asimismo, esta institución, en la nota de prensa sobre el equipamiento y uso de las TIC en los hogares, estimaba que entre los menores de 10 a 15 años el uso de Internet es universal. Por lo que con un margen de error del $4.45 \%$ y un nivel de confianza del $95.5 \%$ se optó por utilizar una muestra de 500 menores. En el trabajo de campo se encuestó a menores de entre 9 y 16, años todos ellos usuarios y usuarias de Internet y a su padre 0 madre. En cada vivienda familiar se entrevistó a aquel de los progenitores que estuviera más implicado en la actividad online del o la menor. Con el fin de maximizar la calidad de las respuestas, los cuestionarios se completaron en los domicilios de las familias y se utilizaron también cuestionarios auto-administrados para las preguntas más sensibles planteadas a los menores.

La muestra se estratificó por región y nivel de urbanización y los puntos de muestreo fueron seleccionados a través de las secciones censales. Posteriormente, las direcciones de las viviendas fueron seleccionadas aleatoriamente a través del procedimiento de rutas aleatorias. La encuesta analizó el acceso y uso de Internet, las actividades online desarrolladas por los menores, la incidencia de los riesgos y la percepción subjetiva del daño ocasionado por los mismos, así como las prácticas comunicativas, las habilidades digitales, aspectos relacionados con el uso excesivo y la mediación parental.

En este artículo vamos a analizar los resultados relacionados con el acoso entre iguales atendiendo al medio a través del cual ha tenido lugar —online y/u offliney a los roles de los menores implicados en los incidentes violentos. El análisis estadístico será fundamentalmente descriptivo ya que el relativamente reducido número de menores victimizados y/0 acosadores no permite hacer análisis estadísticos más elaborados. Los análisis de frecuencias se van a estructurar en función de la edad de los menores puesto que esta influye directamente sobre el desarrollo de habilidades digitales, la maduración de la personalidad, así como la posesión de teléfonos móviles. Se va a analizar la prevalencia del acoso según las diversas modalidades, la frecuencia de los dos roles implicados para el bullying y el ciberbullying, la asociación entre ambos roles y la relación entre daño y edad según el tipo de acoso.

\section{Análisis y resultados \\ 2.1. Contexto y modalidades de cibera- coso}

En la Tabla 1 se muestra cómo la incidencia del bullying cara a cara es muy superior a la del ciberbullying en cualquiera de sus diversas modalidades: 
TABLA 1. Porcentajes de los modos en los que los y las menores han sufrido bullying en los últimos 12 meses según su edad (entre paréntesis, frecuencias absolutas).

\begin{tabular}{|c|c|c|c|c|c|}
\hline \multirow{2}{*}{$\%$} & \multicolumn{4}{|c|}{ Edad } & \multirow{2}{*}{ Total } \\
\hline & $9-10$ & $11-12$ & $13-14$ & $15-16$ & \\
\hline En persona, cara a cara & $27(36)$ & $27(34)$ & $27(34)$ & $20(23)$ & $25(127)$ \\
\hline Llamadas de teléfono móvil & 0 & $1(1)$ & $2(2)$ & $3(3)$ & $1(6)$ \\
\hline $\begin{array}{l}\text { A través de mensajes en mi teléfono } \\
\text { (SMS o MMS) }\end{array}$ & 0 & $1(1)$ & $2(3)$ & $3(3)$ & $1(7)$ \\
\hline En una red social (Tuenti, Facebook) & $1(1)$ & $4(5)$ & $6(7)$ & $8(9)$ & $4(22)$ \\
\hline $\begin{array}{l}\text { En una plataforma (Youtube, Insta- } \\
\text { gram, Flickr) }\end{array}$ & $2(3)$ & $3(4)$ & $6(7)$ & $2(2)$ & $3(16)$ \\
\hline $\begin{array}{l}\text { Por mensajería instantánea (MSN, } \\
\text { WhatsApp, Skype) }\end{array}$ & $2(2)$ & $4(5)$ & $7(9)$ & $9(10)$ & $5(26)$ \\
\hline En un chat & $3(4)$ & $6(7)$ & $4(5)$ & $2(2)$ & $4(18)$ \\
\hline $\begin{array}{l}\text { Alguna forma de bullying a través de } \\
\text { Internet o dispositivos móviles }\end{array}$ & $8(10)$ & $13(16)$ & $14(17)$ & 15 (17) & $12(60)$ \\
\hline TOTAL víctimas & $33(43)$ & $32(40)$ & $33(40)$ & $28(31)$ & $32(154)$ \\
\hline No víctimas & $67(91)$ & $68(87)$ & $67(91)$ & $72(84)$ & $68(346)$ \\
\hline
\end{tabular}

Q33: Si alguien te ha tratado así, ¿cómo ocurrió? (Por favor, marca tantas casillas como sean necesarias).

Base: Todos los y las menores que usan Internet $(\mathrm{N}=500)$.

Fuente: Elaboración propia.

El $12 \%$ de los menores afirma haber sido víctima de ciberacoso, si bien el porcentaje de víctimas de bullying cara a cara (25\%) es más del doble del correspondiente a las víctimas por teléfono o en Internet. Los datos correspondientes a la prevalencia de cada modo muestran que los diversos modos se superponen entre sí. Así, la suma del peso relativo de las víctimas de las diversas modalidades online es superior al porcentaje total de víctimas online (12\%). Del mismo modo que el porcentaje de víctimas cara a cara (25\%) más el online $(12 \%)$ es superior al porcentaje total de víctimas $(32 \%)$ de acoso. Esto demuestra que las modalidades de acoso se superponen unas a otras. Por poner un ejemplo, un mismo niño puede ser acosado cara a cara y por mensajería instantánea y/0 a través de un chat simultáneamente. En cualquier caso, los datos evidencian que el ciberbullying no es el modelo dominante de agresión entre los pares, más bien los datos parecen indicar lo contrario: predomina el acoso cara a cara al que se añaden otras modalidades.

Las vías más habituales a través de las cuales se concreta el ciberbullying son la mensajería instantánea (WhatsApp, 5\%), las redes sociales $(4 \%)$ y los chats $(4 \%)$, estos últimos son utilizados principalmente por los preadolescentes. En cambio, entre los mayores de 13-14 años el acoso online tiene lugar preferentemente a través de mensajería instantánea (7-9\%) 0 en las redes sociales (6-8\%), mientras en el tramo de 13-14 años llama la atención la victimización en las plataformas para compartir $(6 \%)$. 
2.2. Víctimas y acosadores en función de la edad y la modalidad de acoso

Generalmente, cuando se utiliza el término bullying se hace referencia a la victimización de la que son objeto los y las menores. Sin embargo, en este trabajo analizaremos ambas conductas violentas diferenciando los dos roles principales de las personas implicadas: el de la víctima y el del acosador en función de las dos principales modalidades del acoso.

Llama la atención la diferencia entre los diversos grupos de edad: por debajo de los 15 años prevalece claramente el acoso offline (en torno al $27 \%$ en la franja de 9 a 14) frente al 20\% en la franja 15-16 años. En cambio, la incidencia de la victimización a través del ciberbullying tiende a aumentar con la edad, sobre todo a partir de los 11 años, hasta alcanzar el $15 \%$ en el grupo de 15-16 años. En este grupo de edad la diferencia entre las dos modalidades de acoso es del $5 \%$, mientras en los grupos de menor edad es mucho mayor: entre los 11 y 14 años la frecuencia relativa de la victimización offline duplica la correspondiente a la victimización online y entre los más pequeños es tres veces mayor.

En general, la prevalencia del acoso tanto offline como online - es ligeramente inferior a la de la victimización; sin embargo, la distancia entre las frecuencias de los dos roles es más reducida en el caso del ciberbullying. Tanto es así que entre los y las menores de 13-14 años ambas frecuencias son exactamente iguales: en este tramo de edad hay tantas víctimas como acosadores implicados en incidentes de ciberbullying. Las evidencias muestran que la tecnología facilita —así se constató también a través de las entrevistas y grupos de discusión realizados en investigaciones precedentes-el insulto, la venganza, así como el cambio de roles entre los actores implicados.

GRÁFICo 1. Victimización según la edad: bullying vs. ciberbullying.

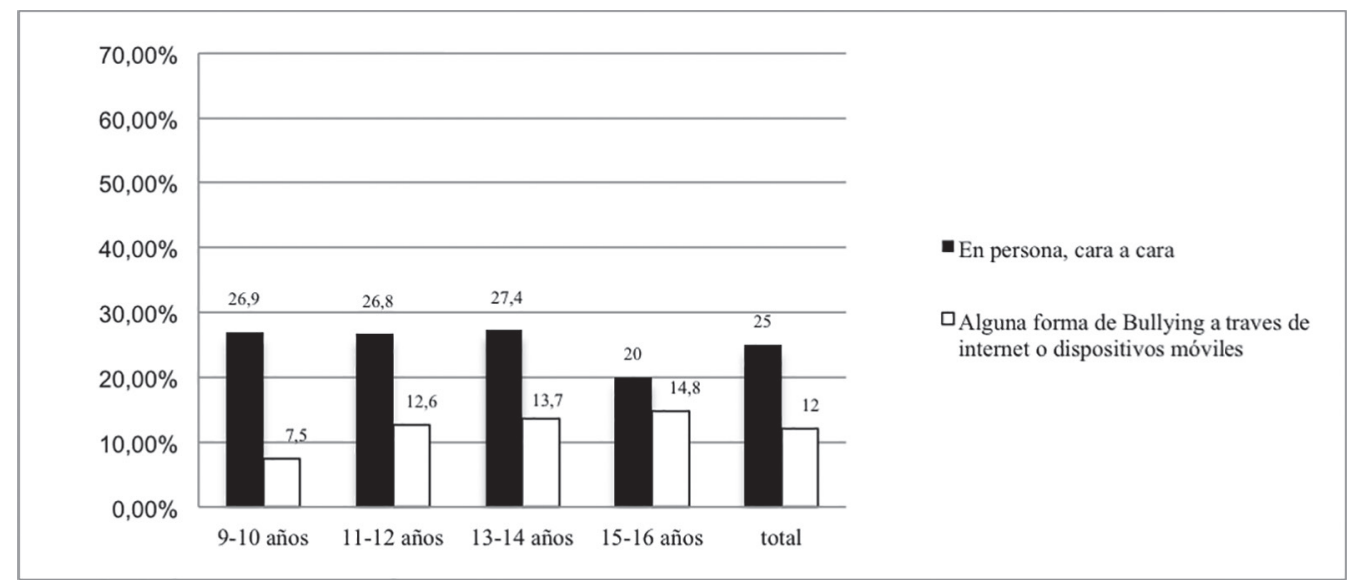

Q33: Si alguien te ha tratado así, ¿cómo ocurrió? (Por favor, marca tantas casillas como sean necesarias).

Base: Todos los y las menores que usan Internet $(\mathrm{N}=500)$.

Fuente: Elaboración propia. 
En general, el porcentaje de acosadores tiende a aumentar con la edad hasta los 13-14 años donde alcanza el $23 \%$ en la modalidad off line y el $14 \%$ online, mientras en la franja de los 15-16 años se reduce hasta el 16\% y el $8 \%$ respectivamente. Ambos tipos de acoso -online y offline - alcanzan su máxima prevalencia en el colectivo de menores de 13-14 años.

GRáfICo 2. Acoso según la edad: bullying vs. ciberbullying.

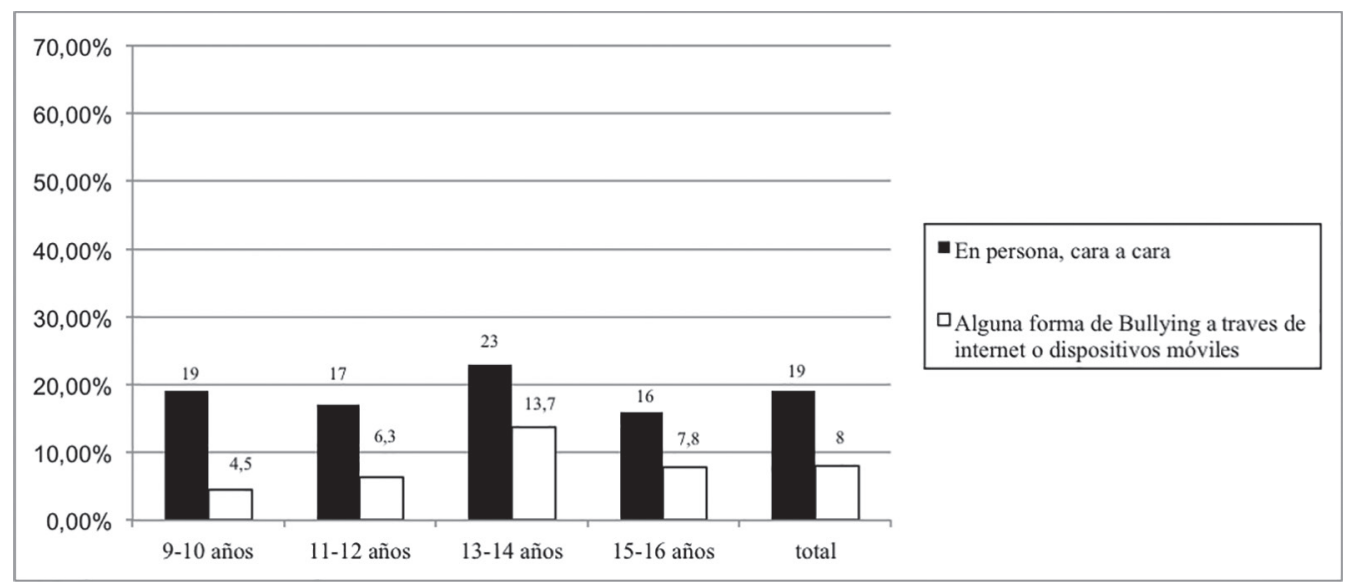

Q34: En los últimos 12 meses, ¿alguna vez te has portado así con alguna otra persona? Si es así, ¿cómo lo hiciste?

(Por favor, marca tantas casillas como sean necesarias). Base: Todos los y las menores que usan Internet $(\mathrm{N}=500)$.

Fuente: elaboración propia.

\subsection{Relación entre agresor y víctima en el entorno online}

La Tabla 2 muestra que hay asociación significativa (Chi cuadrado $=138.5$, significación 0.000) entre los roles de acosador y víctima en el entorno online. En los porcentajes totales el relativamente reducido peso de los y las menores implicados en incidentes de ciberbullying tiende a difuminar las tendencias. No obstante, la participación en episodios de ciberbullying incide enormemente en la probabilidad de ser víctima de acoso. Si por término medio el $12 \%$ de los menores ha sido victimizado, este dato varía sustancialmente dependiendo de que las personas hayan participado como acosadores en incidentes de ciberbullying. Así, entre quienes no han acosado a otros menores online es relativa- mente reducido el porcentaje de los menores que reconocen haber sido víctimas de acoso (7\%), mientras entre los y las menores acosadores tres de cada cuatro han sido a su vez víctimas de acoso online.

TABLA 2. Asociación entre los roles de acosador y víctima en el ciberbullying.

\begin{tabular}{|l|c|c|c|}
\hline \multirow{2}{*}{$\begin{array}{c}\text { \% Víctimas } \\
\text { online }\end{array}$} & \multicolumn{2}{|c|}{ \% Acosadores } & \multirow{2}{*}{ Total } \\
\cline { 2 - 3 } No víctima & $86(428)$ & $2(12)$ & $88(440)$ \\
\hline Víctima & $6(32)$ & $6(28)$ & $12(60)$ \\
\hline Total & $92(460)$ & $8(40)$ & $100(500)$ \\
\hline
\end{tabular}

Q34: En los últimos 12 meses, ialguna vez te has portado así con alguna otra persona? Si es así, ¿cómo lo hiciste? Online.

Q33: Si alguien te ha tratado así, ¿cómo ocurrió? Online.

Base: Todos los y las menores que usan Internet $(\mathrm{N}=500)$.

Fuente: Elaboración propia. 


\subsection{Asociación entre daño y edad se- gún tipo de acoso}

Otro aspecto a tener en cuenta al analizar el bullying y el ciberbullying es el daño que causan a sus víctimas. Investigaciones anteriores avalan que el bullying —online y offline - es en general la experiencia de riesgo más dañina (Livingstone et al., 2011; Mascheroni y Cuman, 2014), respecto a otros riesgos potenciales como el acceso a contenidos inadecuados o el contacto con extraños.

Entre los y las menores víctimas de acoso cara a cara por término medio el $40 \%$ afirmó haberse sentido muy disgustado, el porcentaje correspondiente a quienes manifestaron sentirse un poco disgustados es ligeramente inferior (38.6\%) y el $21 \%$ afirmó no haberse disgustado. Los resultados muestran que la experiencia de daño está fuertemente estructurada por la edad. Así, la proporción de quienes se sintieron muy disgustados aumenta hasta alcanzar el $47 \%$ a los 11 12 años, a los 13-14 años se reduce al $41 \%$ y en el grupo de 15-16 años desciende notablemente situándose en el $22 \%$. La proporción de quienes manifestaron haberse sentido un poco disgustados es ligeramente inferior a la de muy disgustados entre los 9 y los 14 años y a los 15-16 asciende porque se reduce drásticamente el peso relativo de quienes se sintieron muy disgustados. Por último, el porcentaje de quienes no se disgustaron se sitúa en torno al $14 \%$ entre los 9 y los 12 años, aumenta casi 10 puntos a los 13-14 años y se sitúa en el $39 \%$ a los 15-16 años. Los datos evidencian que a partir de los 13 años el desarrollo de la resiliencia y las habili- dades digitales junto con la maduración de la personalidad contribuyen a que los y las menores gestionen más eficazmente las situaciones conflictivas y sean menos vulnerables al daño ocasionado por el acoso cara a cara.

Los resultados evidencian que por término medio la proporción de menores que manifestaron haberse sentido muy disgustados por un episodio de ciberbullying (45\%) es superior a la de quienes se sintieron así a causa del bullying cara a cara (40\%). En cambio, el porcentaje de menores que afirmó haberse sentido un poco disgustados es inferior en el caso del ciberbullying (28\% frente al $39 \%$ ). Los datos muestran que la percepción subjetiva del daño causado por ciberbullying está más polarizada: son relativamente más numerosos quienes han estado muy disgustados, pero son también más numerosos quienes afirman no haberse sentido afectados.

En cuanto a la edad, llama la atención que entre los menores de 12 años el porcentaje de quienes se sintieron muy disgustados se sitúa entre el $40 \%$ y el $44 \%$, frente al $30 \%$ que dice que no le afectó el ciberbullying. Este último dato podría indicar que a esa temprana edad algo menos de uno de cada tres niños ya está desarrollando su resiliencia. Sin embargo, los datos del grupo de 13-14 años son totalmente contrapuestos. La vulnerabilidad de este grupo a la victimización online es muy notable: el $65 \%$ afirma que se sintió muy disgustado, el $24 \%$ algo disgustado y tan solo el $12 \%$ no se disgustó. Posiblemente, la altísima vulnerabilidad de este 
tramo de edad al ciberbullying esté asociada a la importancia que estos menores atribuyen a su reputación online, que se verá seriamente afectada por los incidentes de violencia online. En cambio, entre los y las menores de 15-16 años se reduce notablemente el porcentaje de quienes se sintieron muy disgustados (29\%), al igual que ocurre en el caso del bullying, y los porcentajes de quienes se sintieron algo disgustados y no se disgustaron se equipa$\operatorname{ran}(35 \%)$.

GRáfICo 3. Nivel de daño entre las víctimas de bullying cara a cara.

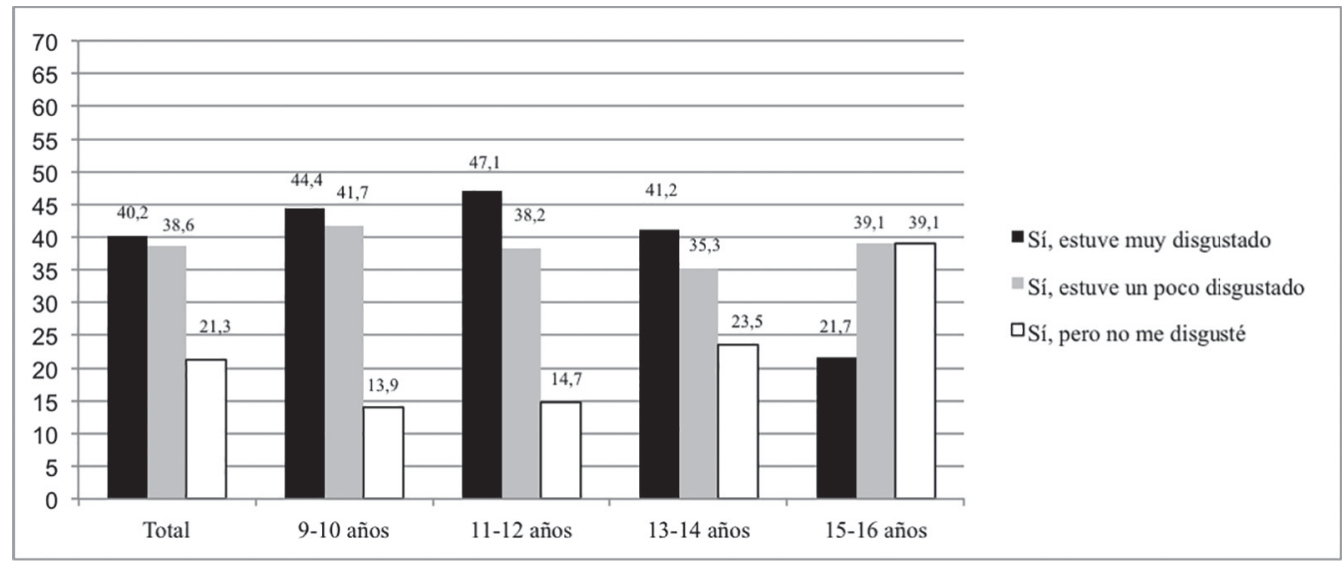

Q32: En los últimos 12 meses, ¿alguien te ha tratado así? Si es así, ¿cómo estabas de disgustado/a? Base: Todos los y las menores que usan Internet y han sido víctimas de bullying cara a cara

N

$m$

(2)

กิ

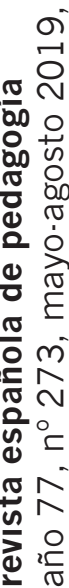
$(\mathrm{N}=127)$.

Fuente: Elaboración propia.

GRÁFICO 4. Nivel de daño entre las víctimas de ciberbullying.

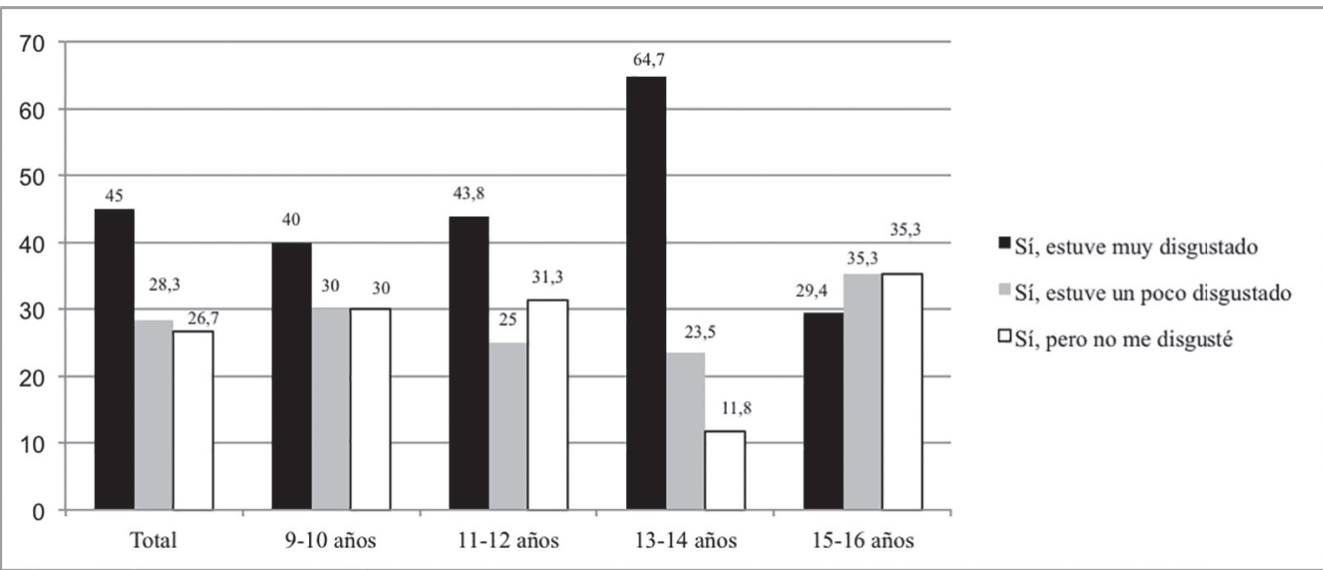

Q32: En los últimos 12 meses, ¿alguien te ha tratado así? Si es así, ¿cómo estabas de disgustado/a? Base: Todos los y las menores que usan Internet y han sido víctimas de ciberbullying $(\mathrm{N}=60)$.

Fuente: Elaboración propia. 


\section{Discusión}

Las evidencias demuestran que las situaciones de acoso online tienen lugar en un contexto en el que existen muchos más casos de acoso cara a cara: el acoso online o ciberacoso es uno más, que se superpone a otros tipos de acoso ya existentes, lo cual coincide con lo constatado por investigaciones y revisiones precedentes (Ybarra, Boyd, Korchmaros y Oppenheim, 2012; Zych et al., 2015; Garmendia et al., 2016). La evolución de ambos tipos de acoso mantiene una tendencia creciente, así la prevalencia del acoso en general -bien sea online u offline- se ha duplicado con respecto a la del año 2010; el 15\% de los menores de 9 a 16 años (Garmendia, Garitaonandia, Martínez y Casado, 2011) frente al $32 \%$ en 2015. Este notable incremento en la incidencia del acoso puede estar relacionado con una mayor facilidad para identificar situaciones de abuso y acoso por parte de quienes las sufren y con una mayor conciencia social en torno al mismo (Garmendia et al., 2018).

Respecto a la relación entre la prevalencia del ciberbullying y la edad, si bien Del Rey et al. (2018) detectan resultados dispares, en nuestro trabajo las diferencias en función de los grupos de edad evidencian una progresión de los comportamientos violentos online entre iguales: la frecuencia del ciberbullying aumenta a medida que lo hace la edad a diferencia de lo que ocurre con el bullying cara a cara (González-Calatayud, 2018; Barboza, 2015; Cappadocia, Craig y Peppler, 2013; Kowalski y Limber, 2007). Son más las victimas que sufren el ciberacoso conforme van aumentando en edad, aunque estos comportamientos de acoso sean mantenidos por menos alumnos. Luego, un menor número de agresores acosan y victimizan a mayor número de alumnos en los últimos cursos de secundaria. Sin embargo, en el acoso offline la frecuencia aumenta con la edad, pero a partir de los 15 años se constata un cambio de tendencia, reduciéndose el acoso cara a cara.

La prevalencia de las conductas de acoso en el grupo de 13-14 años es llamativamente elevada. Esto coincide con el primer ciclo de la enseñanza secundaria, donde la supervisión de los estudiantes en los centros escolares es relativamente menor y al mismo tiempo también aumenta de modo notable la posesión de teléfonos móviles (INE, 2017) con frecuencia asociada a un rito de paso - en este sentido, es frecuente entre los menores recibir un smartphone de regalo con ocasión de su paso a la Educación Secundaria- (Mascheroni y $\mathrm{Cu}$ man, 2014). En cambio, a partir de los 15-16 años desciende notablemente la prevalencia del acoso cara a cara, que puede ser interpretada como parte del proceso de maduración y aprendizaje de la gestión de las emociones y las consecuencias de los actos.

En este sentido, es importante tener en cuenta que el anonimato, y la facilidad de uso y la inmediatez contribuyen a que insultar online sea percibido como fácil y exento de consecuencias. Por consiguiente, las evidencias pueden entenderse como una banalización de la violencia online. En muchas ocasiones el contexto de la agresión está relacionado con parejas de jóvenes que en una gestión inmadura de la 
ruptura difunden mensajes 0 imágenes de la expareja como chantaje 0 venganza para dañar su reputación (Roca, 2015), lo cual resulta consistente respecto a la asociación que plantea Görzig (2011) entre el ciberbullying, la búsqueda de sensaciones y las dificultades asociadas a la maduración. Como conclusión a este respecto, y vista la evolución del ciberbullying por franjas de edad, se deduce que prestar especial atención al primer ciclo de educación secundaria, cuando coinciden una incorporación casi universal al smartphone y una falta de habilidad para gestionar las relaciones sociales, resulta vital para prevenir y abordar situaciones de acoso online. En esta línea, Garaigordobil defiende que «si no se interviene de forma preventiva es esperable que el ciberbullying no solo no disminuya sino que aumente entre los 12 y los 18 años» (2015, p. 1074), entendiendo que son fundamentales unos buenos hábitos y planes de prevención del ciberacoso para aprender a relacionarse online.

Respecto a la relación entre los roles de acosador y víctima online, existe una asociación significativa entre ambos. Los datos no permiten esclarecer cuál es la secuencia en el intercambio de los dos roles, si la victimización promueve las conductas de acoso o si son las conductas de acoso las que provocan la victimización posterior de los acosadores. No obstante, son numerosas las evidencias de investigaciones anteriores que avalan esta asociación, como la de Görzig (op. cit.), que demostró que en torno al $60 \%$ de los acosadores había sido también acosado, y la de Lampert y Donoso, que también señalaron que «ser un ciberacosador es el mejor predictor de ser víctima de ciberbullying» y las fronteras entre roles son más difíciles de trazar en el ciberbullying que en el acoso cara a cara (2012, p. 146).

Respecto a la incidencia del daño, entendiéndola como una experiencia subjetiva que puede ser diferente en cada experiencia de acoso, los datos muestran que la percepción subjetiva del daño causado por ciberbullying está más polarizada que en el caso del bullying cara a cara: son relativamente más numerosos quienes expresan estar muy disgustados al respecto, pero son también más numerosos quienes afirman no haberse sentido afectados por ello. También se percibe una fuerte estructuración por edad: a medida que aumenta la edad de las víctimas se reduce la proporción de daño severo, pero este proceso es algo diferente entre el bullying cara a cara y el online. A partir de los 13 años el desarrollo de la resiliencia y la maduración pueden contribuir a que los y las menores gestionen con mayor eficacia los conflictos y sean menos vulnerables al daño ocasionado por el acoso convencional. Y, sin embargo, en la franja de 13-14 años la vulnerabilidad ante el ciberbullying es extraordinaria: el $65 \%$ de los afectados afirmó sentirse muy disgustado, el $24 \%$ algo disgustado y tan solo el $12 \%$ no se disgustó. Como se ha indicado, esta altísima vulnerabilidad está probablemente asociada a la importancia de la reputación online en esta franja de edad, y a la percepción de que la agresión puede tener un alcance universal (Ruiz, Martín, López y Hernán, 2016), lo cual redunda en el interés particular de las tareas de prevención y gestión de conflictos entre estos menores. 


\section{Conclusiones}

La estrecha asociación señalada en nuestro trabajo entre los roles de acosador y victimizado indican que el clima escolar se ve afectado por situaciones de violencia, en las que surge y que a su vez son alimentadas por los episodios de agresiones, y en las cuales también participan aquellos niños y niñas que observan, sufren y/o reproducen. Todo esto hace que la intervención escolar sea necesaria para proteger a la víctima y reeducar al agresor (Save the Children, 2016), y además constituye un importante elemento unificador al margen de la situación de partida de cada familia respecto, por ejemplo, a las habilidades digitales (Garmendia et al., 2016). Actuar en los centros escolares implica dificultades como la ley del silencio escolar en virtud de la cual el profesorado no puede ayudar a las víctimas porque no siempre es consciente de lo que está sucediendo (Del Rey et al., 2018), o la falta de preparación específica para gestionar la situación. En este sentido, es importante detectar situaciones de acoso, e igualmente disponer de protocolos de actuación y planes de prevención, tomando como referencia aquellas claves demostradamente válidas contra el bullying tradicional (Ttofi y Farrington, 2011), pero también otras específicas, dadas la diferencias detectadas entre ambos tipos de agresión.

El Defensor del Pueblo recomienda que los centros educativos tengan cada vez mayor intervención sobre el acoso, trabajando con familias y orientadores, y dotando al alumnado de habilidades de relación interpersonal y estrategias de comunicación. Esto es, resolviendo los temas de convivencia dentro de la misma escuela, sin acudir tanto a intervenciones externas, servicios sociales, policía o jueces (Defensor del Pueblo, 2007). En este sentido, hay evidencias que avalan que en los centros educativos con proyecto de educación para la solución de los conflictos, disminuye el número de problemas en las relaciones entre alumnos y alumnas (Caballero, 2010).

Pero la realidad dista enormemente de lo que se prescribía, los recortes ocasionados por la crisis han supuesto que en muchos centros escolares ya no se pueda contar con la figura de apoyo a la convivencia del PTSC (Profesorado Técnico de Servicios a la Comunidad) que en teoría sería la persona responsable de la implantación de los protocolos o planes contra el acoso escolar en los centros, lo cual, a la luz de las evidencias aquí reproducidas, constituye un grave error. Así, llama la atención que «en las menciones que se hacen en la LOMCE sobre formación para profesorado y concesión de medios a centros, se relaciona de forma constante la calidad educativa con las asignaturas curriculares, no considerándose esencial la prevención de la violencia como parte de esa calidad educativa a la que se aspira» (Del Rey et al., 2018, p.86). Simultáneamente, el respaldo normativo a la necesidad de afrontar la violencia escolar es todavía escaso y está poco ajustado a las indicaciones tanto del Defensor del Pueblo como de los principales investigadores sobre la temática en nuestro entorno, y sigue siendo imprescindible crear legislaciones específicas relativas a la violencia escolar (Cerezo y Rubio, 2017). En suma, resulta preocupante el creciente interés social por el maltrato entre iguales frente a la ausencia de implicación política para prevenirlo. 
En este sentido, el informe "Los derechos de los niños y niñas en el sistema educativo en España» (Larrañaga, 2016) destaca la aceptación social de la violencia en algunos entornos escolares donde los agresores tienden a replicar patrones de violencia en la relación con sus pares sin llegar a ser conscientes del daño causado a la víctima. Es evidente que el entorno escolar es altamente permeable a la violencia en otros entornos sociales de la sociedad actual, que se caracteriza por el individualismo, la competitividad y una vida acelerada donde las relaciones interpersonales se vuelven cada vez más conflictivas. La recuperación de la convivencia escolar es un reto que debe implicar a todos los actores — profesorado, alumnado y progenitores- para transformar la cultura del mal trato en cultura del buen trato.

Asimismo, se ha probado que un clima amigable y de apoyo en la escuela tiene efectos positivos sobre los y las estudiantes y reduce la probabilidad de acoso tanto online como cara a cara. La investigación muestra que cuando los jóvenes se sienten conectados a sus escuelas, en un clima de confianza, imparcial, agradable y positivo, menor es la implicación reconocida en todo tipo de acoso - físico, verbal o en Internet- (Cohen-Almagor, 2018; Cohen, Twemlow, Berkowitz y Comer, 2015; Williams y Guerra, 2007).

Las escuelas tienen pues la responsabilidad de actuar ante el acoso, bien sea virtual o cara a cara, exponiendo y debatiendo sobre el problema en el centro escolar, tratando de explicar los efectos que tiene el bullying sobre las víctimas. Pero el tra- bajo de las escuelas se ha desarrollar junto con los padres con el fin de que se tome conciencia y se controle el problema. Son necesarios programas de prevención en la escuela, que ofrezcan apoyo a los alumnos, pero también es necesaria la intervención parental (Cohen-Almagor, 2018).

Y las escuelas no pueden ellas solas abordar esta problemática social. Las últimas investigaciones sobre la temática sugieren que hacen falta tomar medidas ante los males sociales que genera Internet, y se reclama una cooperación responsable entre padres, escuelas, gobiernos o instituciones, organizaciones no gubernamentales (ONG) y los responsables de las redes sociales. La intimidación es un problema que nos afecta y nos preocupa a todos, por lo que hay que abordarlo de forma interdisciplinar. La responsabilidad y la rendición de cuentas deben ser compartidas por todas las partes: padres, maestros, escuelas, organizaciones no gubernamentales y empresas, y abarcar diferentes países o la comunidad internacional (Cohen-Almagor, 2018).

\section{Referencias bibliográficas}

Barboza, G. E. (2015). The association between school exclusion, delinquency and subtypes of cyber - and F2F - victimizations: Identifying and predicting risk profiles and subtypes using latent class analysis. Child Abuse \& Neglect, 39, 109-122. doi: https://doi.org/10.1016/j. chiabu.2014.08.007

Caballero, M. J. (2010). Un estudio sobre buenas prácticas. Revista paz y conflictos, 3, 154-169.

Cappadocia, M. C., Craig, W. M. y Pepler, D. (2013). Cyberbullying prevalence, stability, and risk factors during adolescence. Canadian Journal of School Psychology, 28 (2), 171-192. doi: https://doi.org/10.1177/0829573513491212 
Casas, J. A., Del Rey, R. y Ortega-Ruiz, R. (2013). Bullying and cyberbullying: convergent and divergent predictor variables. Computers in $\mathrm{Hu}$ man Behaviour, 29 (3), 580-587. doi: https://doi. org/10.1016/j.chb.2012.11.015

Cerezo, F. y Rubio, F. J. (2017). Medidas relativas al acoso escolar y ciberacoso en la normativa autonómica española. Un estudio comparativo. Revista Electrónica Interuniversitaria de Formación del Profesorado, 20 (1), 113-126.

Cohen, J., Espelage, D. L., Twemlow, S. W., Berkowitz, M. W. y Comer, J. P. (2015). Rethinking effective bully and violence prevention efforts: Promoting healthy school climates, positive youth development, and preventive bully-victim-bystander behavior. International Journal of Violence \& Schools, 15 (1), 2-40.

Cohen-Almagor, R. (2018). Social responsibility on the Internet: Addressing the challenge of cyberbullying. Aggression and Violent Behavior, 39, 42-52. doi: https://doi.org/10.1016/j. avb.2018.01.001

Comisión Europea (2015). Special Eurobarometer 423: Cyber Security. Bruselas: Unión Europea.

Cook, C. R., Williams, K. R., Guerra, N. G., Kim, T. E. y Sadek, S. (2010). Predictors of bullying and victimization in childhood and adolescence: a meta-analytic investigation. School Psychology Quarterly, 25 (2), 65-83. doi: http:// dx.doi.org/10.1037/a0020149

Currie, C., Zanotti, C., Morgan, A., Currie, D., De Looze, M. y Roberts, C. (2012). Health policy for children and adolescents (Report n.6). Social determinants of health and well-being among young people: Health Behaviour in School-aged Children (HBSC) study: International report from the 2009/2010 survey. Copenhage: World Health Organization, Regional Office for Europe.

Defensor del Pueblo (2007). Violencia escolar: el maltrato entre iguales en la educación secundaria obligatoria 1999-2006. Madrid: Defensor del Pueblo.

Del Rey, R., Estévez, M., Ojeda, M. (2018). El ciberbullying y su respuesta educativa. En E. Jiménez, M. Garmendia y M. A. Casado, Entre selfies y whatsapps. Oportunidades y riesgos para la infancia y la adolescencia conectada (pp. 125-139). Barcelona: Gedisa.
Del Rey, Elipe y Ortega-Ruiz (2012). Bullying and cyberbullying: overlapping and predictive value of the co-occurrence. Psicothema, 24 (4), 608-613.

Estévez, A., Villardón, L., Calvete, E., Padilla P. y Orue, I. (2010). Adolescentes víctimas de cyberbullying: prevalencia y características. Behavioral Psychology/Psicología Conductual, 18 (1), 73-89.

Garaigordobil, M. (2011). Prevalencia y consecuencias del cyberbullying: una revisión. International Journal of Psychology and Psychological Therapy, 11 (2), 233-254.

Garaigordobil, M. (2015). Ciberbullying en adolescentes y jóvenes del País Vasco: Cambios con la edad. Anales de psicología, 31 (3), 1069-1076. doi: https://doi.org/10.6018/ analesps.31.3.179151

Garmendia, M., Garitaonandia, C., Martínez, G., Casado, M. A. (2011). Riesgos y seguridad en Internet: Los menores españoles en el contexto europeo. Bilbao: EU Kids Online, Universidad del País Vasco / Euskal Herriko Unibertsitatea. Recuperado de http://www.lse.ac.uk/media@ lse/research/EUKidsOnline/EU\%20Kids\%20 II\%20(2009-11)/National\%20reports/Spanish\%20report.pdf (Consultado el 29/10/2018).

Garmendia, M., Jiménez, E., Casado, M. A. y Mascheroni, G. (2016). Net Children Go Mobile: Riesgos y oportunidades en Internet y el uso de dispositivos móviles entre menores españoles (2010-2015). Madrid: Red.es / Universidad del País Vasco / Euskal Herriko Unibertsitatea. Recuperado de https://addi.ehu.es/ handle/10810/21546 (Consultado el 29/10/2018).

Garmendia, M., Casado, M. A., Jiménez, E. y Garitaonandia, C. (2018). Oportunidades, riesgos, daño y habilidades digitales de los menores españoles. En E. Jiménez, M. Garmendia y M. A. Casado, Entre selfies y whatsapps. Oportunidades y riesgos para la infancia y la adolescencia conectada (pp.31-54). Barcelona: Gedisa.

González-Calatayud, V. (2018). Victims of cyberbullying in the Region of Murcia: a growing reality. Journal of New Approaches in Educational Research, 7 (1), 10-16.

Görzig, A. (2011). Who bullies and who is bullied Online?: a study of 9-16 year old Internet users in 25 European countries. Londres: EU Kids Online network. Recuperado de http://eprints. lse.ac.uk/39601 (Consultado el 29/10/2018). 
Hinduja, S. y Patchin, J. W. (2008). Cyberbullying: an exploratory analysis of factors related to offending and victimization. Deviant Behavior, 29 (2), 129-156. doi: https://doi. org $/ 10.1080 / 01639620701457816$

Hinduja, S. y Patchin, J. W. (2009). Bullying beyond the schoolyard: Preventing and responding cyberbullying. Thousand Oaks, CA: Sage Publications.

Inchley, J., Currie, D., Young, T., Oddrun, S. y Torbjorn, T. (2016). Health policy for children and adolescents, no. 7. Growing up unequal: gender and socioeconomic differences in young people's health and well-being. Health Behavior in Schoolaged Children (HSB) Study: International report from the 2013/2014 survey. Copenhage: World Health Organization, Regional Office for Europe.

Instituto Nacional de Estadística (INE) (2017). Encuesta sobre Equipamiento y Uso de Tecnologías de Información y Comunicación en los Hogares 2017. Madrid: Instituto Nacional de Estadística.

Instituto Vasco de Evaluación e Investigación Educativa (ISEI-IVEI) (2017). Maltrato entre iguales en Euskadi 2016. Bilbao: Gobierno Vasco. Departamento de Educación. Recuperado de https://bit.ly/2U7Ivhp (Consultado el 09/04/2019).

Kowalski, R. M. y Limber, S. P. (2007). Electronic Bullying Among Middle School Students. Journal of Adolescent Health, 41 (6), 22-30. doi: https:// doi.org/10.1016/j.jadohealth.2007.08.017

Lampert, C. y Donoso, V. (2012). Bullying. En S., Livingstone, L. Haddon y A. Görzig (Eds.), Children risk and safety on the Internet (pp.139148). Bristol: Policy Press.

Larrañaga, K. P. (2016). Los derechos de los niños y niñas en el sistema educativo en España. Análisis de la situación. Barcelona: Asociación GSIA / Fundación Educo. Recuperado el de http:// www.bienestaryproteccioninfantil.es/fuentes1.asp? sec $=10 \&$ subs $=25 \& \operatorname{cod}=3460 \&$ page $=\& \mathrm{v}=2$ (Consultado el 29/10/2018).

Livingstone, S., Haddon, L., Görzig, A. y Ólafsson, K. (2011). Risk and safety on the Internet. The perspective of European children. Full Findings. Londres: EU Kids Online, The London School of Economics and Political Science (LSE).
Levy, N., Cortesi, S., Crowley, E., Beaton, M., Casey, J. y Nolan, C. (2012). Bullying in a networked era: A literature review. Berkman Center Research Publication, 2012-17. Recuperado de http:// cyber.law.harvard.edu/publications/2012/kbw bulling in a networked_era (Consultado el 29/10/2018).

López-Pradas, I. C., Romera, E. M., Casas, J. A. y Ortega-Ruiz, R. (2017). Cybergossip and cyberbullying during primary school years. Psicología Educativa, 23 (2), 73-80. doi: https://doi. org/10.1016/j.pse.2017.05.007

Mascheroni, G. y Cuman, A. (2014). Net Children Go Mobile: Final report. Deliverables D6.4\&D5.2. Milano: Educatt.

Menesini, E., Nocentini, A., Palladino, B. E., Frisén, A., Berne, S., Ortega-Ruiz, R., ... Smith, P. K. (2012). Cyberbullying definition among adolescents: a comparison across six European countries. Cyberpsychology, behavior, and social networking, 15 (9), 455-463. doi: https:// doi.org/10.1089/cyber.2012.0040

Ortega, R., Calmaestra, J. y Mora, J. (2008). Cyberbullying. International Journal of Psychology and Psychological Therapy, 8 (2), 183-192.

Ovejero, A., Smith, P. K. y Yubero, S. (Coords.) (2013). El acoso escolar y su prevención: perspectivas internacionales. Madrid: Biblioteca Nueva.

Roca, G. (2015). Las nuevas tecnologías en niños y adolescentes. Guía para educar saludablemente en una sociedad digital. Barcelona: Hospital Sant Joan de Déu.

Rubio Sáiz, M. (2013). Estudio sobre la percepción del profesorado en educación secundaria obligatoria del acoso escolar. Revista de Educación Social, 16. Recuperado de http://www.eduso. net/res/pdf/16/acos_res_\%2016.pdf (Consultado el 09/04/2019).

Ruiz Benítez, B., Martín Barato, A., López Catalán, B. y Hernán García, M. (2016). ¿Convivencia 0 Bullying?: análisis, prevención y afrontamiento del acoso entre iguales. Granada: Consejería de Salud. Escuela Andaluza de Salud Pública.

Sahuquillo Verdet, F. (2017). Fuentes y encuadres del discurso mediático del acoso escolar en los periódicos El Mundo y El País. Doxa Comunicación, 25, 169-192. 
Save the Children (2016). Yo a eso no juego. Bullying y cyberbullying en la infancia. Recuperado de https://www.savethechildren.es/sites/ default/files/imce/docs/yo_a_eso_no_juego.pdf (Consultado el 29/10/2018).

Slonje, R., Smith, P. K. y Frisén, A. (2013). The nature of cyberbullying, and strategies for prevention. Computers in Human Behavior, 29 (1), 26-32. doi: https://doi.org/10.1016/j. chb.2012.05.024

Smith, P. K. (2016). Bullying escolar. En S. Yubero, E. Larrañaga, R. Navarro (Eds.), La violencia en las relaciones humanas: contextos y entornos protectores del menor (pp.9-29). Cuenca: Ediciones de la Universidad de Castilla-La Mancha.

Smith, P. K., Kwak, K. y Toda, Y. (2016). School bullying in different cultures. Cambridge: Cambridge University Press.

Ttofi, M. M. y Farrington. D. P. (2011). Effectiveness of school-based programs to reduce bullying: a systematic and meta-analytic review. Journal of Experimental Criminology, 7 (1). 27-56. doi: https://doi.org/10.1007/s11292-0109109-1

Williams, K. R. y Guerra, N. G. (2007). Prevalence and predictors of Internet bullying. The Journal of Adolescent Health, 41 (6), S14-S21.

Ybarra, M. L., Boyd, D., Korchmaros, J. D. y Oppenheim, J. (2012). Defining and measuring cyberbullying within the larger context of bullying victimization. Journal of Adolescent Health, 51 (1), 53-58. doi: https://doi.org/10.1016/j. jadohealth.2011.12.031

Zych, I., Ortega-Ruiz, R. y del Rey, R. (2015). Systematic review of theoretical studies on bullying and cyberbullying: facts, knowledge, prevention, and intervention. Aggression and Violent Behaviour, 23, 1-21. doi: https:/doi. org/10.1016/j.avb.2015.10.001

\section{Biografía de las autoras}

Maialen Garmendia Larrañaga es

Doctora en Ciencias Políticas y Sociología por la Universidad de Deusto. Profesora Titular de Universidad del Departamento de Sociología y Trabajo Social de la Uni- versidad del País Vasco UPV/EHU. Su actividad investigadora se ha centrado en torno a las tecnologías de la comunicación, la vida cotidiana, los menores y las cuestiones de género. Desde el año 2006 forma parte de la red de investigación EU Kids Online.

(D) https://orcid.org/0000-0002-8057-6370

Estefanía Jiménez Iglesias es Doctora en Comunicación Audiovisual por la Universidad del País Vasco UPV/EHU. Profesora Agregada en el Departamento de Comunicación Audiovisual y Publicidad de la UPV/EHU. Sus trabajos de investigación se centran en la vida digital de la infancia y la adolescencia. Forma parte de la red EU Kids Online desde 2012.

(D) https://orcid.org/0000-0003-2897-5929

Nekane Larrañaga Aizpuru es Doctora en Ciencias Políticas y Sociología por la Universidad de Deusto. Profesora Agregada en el Departamento de Sociología y Trabajo Social en la Universidad del País Vasco UPV/EHU. Su actividad investigadora se ha centrado en torno a la diversidad, las relaciones interculturales e interétnicas, educación y valores, bilingüismo, identidad, ciudadanía 0 aculturación.

(D) https://orcid.org/0000-0002-8062-3544 


\section{revista española de pedagogía \\ año 77, n 273, mayo-agosto 2019 \\ Spanish Journal of Pedagogy \\ year 77, n. 273, May-August 2019 \\ Sumario* \\ Table of Contents **}

\section{Respuestas educativas \\ y cívico-penales a los comportamientos antisociales}

\section{Educational and civic-penal responses to antisocial behavior}

Editor invitado: Fernando Gil Cantero Guest editor: Fernando Gil Cantero

\section{Fernando Gil Cantero}

Presentación: Respuestas educativas y cívico.penales a los comportamientos antisociales

Introduction: Educational and civic-penal responses to antisocial behavior

David Reyero, Fernando Gil Cantero

La educación que limita es la que libera

Education that limits is education that frees

Maria José Bernuz Beneitez, Esther Fernández Molina La pedagogía de la justicia de menores: sobre una justicia adaptada a los menores

The pedagogy of juvenile justice: a child-friendly justice

Marina Martins, Carolina Carvalho

¿En qué mienten los adolescentes?

What do teenagers lie about?
Inmaculada Méndez, Cecilia Ruiz Esteban, Juan Pedro Martínez, Fuensanta Cerezo

Ciberacoso según características sociodemográficas y académicas en estudiantes universitarios

Cyberbullying according to sociodemographic and academic characteristics among university students

261

Irene Montiel, José R. Agustina

Retos educativos ante los riesgos emergentes en el ciberespacio: claves para una adecuada prevención de la cibervictimización en menores

Educational challenges of emerging risks in cyberspace: foundations of an appropriate strategy for preventing online child victimization

Maialen Garmendia Larrañaga, Estefanía Jiménez Iglesias, Nekane Larrañaga Aizpuru Bullying y ciberbullying: victimización, acoso y daño.

Necesidad de intervenir en el entorno escolar Bullying and cyberbullying: victimisation, harassment, and harm. The need to intervene in the educational centre

Ana Rosser-Limiñana, Raquel Surià-Martínez Adaptación escolar y problemas comportamentales y emocionales en menores expuestos a violencia de género

School adaptation and behavioural and emotional problems in minors exposed to gender violence

\footnotetext{
* Todos los artículos están también publicados en inglés en la página web de la revista: https://revistadepedagogia.org.

** All the articles are also published in English on the web page of the journal: https://revistadepedagogia.org.
} 
Fanny T. Añaños-Bedriñana, Miguel Melendro, Rocío

Raya Miranda

Mujeres jóvenes con medidas de protección y judiciales y sus tránsitos hacia la prisión

Young women with protective and judicial measures and their transit towards prison

333

\section{Reseñas bibliográficas}

\section{Esteban Bara, F. Ética del profesorado (Juan} García Gutiérrez). García Amilburu, M., Bernal, A. y González Martín, M. R. Antropología de la educación. La especie educable (Yaiza Sánchez Pérez). Rose, D. y Martin, J. R. Leer para aprender. Lectura y escritura en las áreas del currículo (Francisco Lorenzo Bergillos). Buxarrais, M. R. y Vilafranca, I. (Coords.). Una mirada femenina de la educación moral (Eric Ortega González).

351

\section{Informaciones}

«ECER conference Education in an Era of Risk.the Role of Educational Research for the Futurey - de la European Educational Research Association (EERA)-; III Congreso «European Liberal Arts and Core Texts Educations; VIII Congreso del Jubilee Centre for Character and Virtues: «Virtues and the Flourishing Lifen; XLV Congreso Internacional de AME 2019: «Morality and Ethics for the Digital World»; III Premio Ricardo Marín Ibáñez; Una visita a la hemeroteca (profesores youtubers) (Daniel Pattier); Una visita a la red (David Reyero).

\section{Instrucciones para los autores}

Instructions for authors

375

ISSN: 0034-9461 (Impreso), 2174-0909 (Online)

https://revistadepedagogia.org/

Depósito legal: M. 6.020 - 1958

INDUSTRIA GRÁFICA ANZOS, S.L. Fuenlabrada - Madrid 


\title{
Bullying and cyberbullying: victimisation, harassment, and harm. The need to intervene in the educational centre
}

\section{Bullying y ciberbullying: victimización, acoso y daño. Necesidad de intervenir en el entorno escolar}

\begin{abstract}
Maialen GARMENDIA LARRAÑAGA, PhD. Senior Lecturer. Universidad del País Vasco (maialen.garmendia@ehu.eus). Estefania JIMÉNEZ IGLESIAS, PhD. Senior Lecturer. Universidad del País Vasco (estefania.jimenez@ehu.eus). Nekane LARRAÑAGA AIZPURU, PhD. Senior Lecturer. Universidad del País Vasco (nekane.larranaga@ehu.eus).
\end{abstract}

\section{Abstract:}

This work focusses on cyberbullying, analysing the results of a survey administered to a representative sample of Spanish internet users aged between 9 and 16 . The data show that harassment on digital devices is part of the climate of violence among pre-teenagers and teenagers, where face-to-face bullying is much higher than online bullying. Although bullying occurs in different ways, and these tend to overlap, the most frequent form is offline bullying. The prevalence of cyberbullying varies considerably by age, tending to increase as the subjects' age increases, whereas offline bullying decreases among 15-16-year-olds. The boundary between victims and perpetrators is difficult to sketch in cyberbullying as three out of four children who admit having treated others in a hurtful or nasty way on the Internet or with mobile phones have themselves been treated in this way by others. The evidence regarding 13-14-year-olds is especially worrying as they are more involved in cyberbullying and a great many of them say they have felt very upset when victimized. The results display a need to prevent and deal with cyberbullying at school, as this is the most effective and equitable site for intervention.

Keywords: bullying, teenagers, cyberbullying, school life, Internet, mobile devices, risks.

Revision accepted: 2019-03-03.

This is the English version of an article originally printed in Spanish in issue 273 of the revista española de pedagogía. For this reason, the abbreviation EV has been added to the page numbers. Please, cite this article as follows: Garmendia Larrañaga, M., Jiménez Iglesias., E., \& Larrañaga Aizpuru, N. (2019). Bullying y ciberbullying: victimización, acoso y daño. Necesidad de intervenir en el entorno escolar | Bullying and cyberbullying: victimisation, harassment, and harm. The need to intervene in the educational centre. Revista Española de Pedagogía, 77 (273), 295-312. doi: https://doi.org/10.22550/REP77-2-2019-08 


\section{Resumen:}

El presente trabajo analiza el ciberbullying a través de los resultados de una encuesta realizada a una muestra representativa de los y las menores españolas usuarias de Internet de entre 9 y 16 años. Los datos revelan que el acoso a través de las tecnologías digitales forma parte de un clima de violencia entre los preadolescente y adolescentes, entre los cuales el acoso cara a cara prevalece claramente sobre el que tiene lugar a través de medios tecnológicos. Si bien las diversas modalidades de acoso se superponen entre sí, la más frecuente es la que tiene lugar en entornos offline. La incidencia del ciberbullying está fuertemente estructurada por la edad y aumenta en función de esta. Se aprecia un intercambio de roles entre los y las menores implicados en los episodios violentos online, dado que tres de cada cuatro acosadores han sido también víctimas de acoso. Resultan particularmente llamativos los datos del grupo de 13-14 años, en el que la prevalencia de la participación en el ciberbullying es relativamente elevada y además los y las menores reconocen haberse sentido muy disgustados por su victimización. Los resultados avalan la necesidad de intervenir desde el entorno escolar para minimizar la incidencia del acoso, ya que es el principal ámbito en el que tiene lugar, y además este es el ámbito más igualitario y efectivo.

Palabras clave: acoso, adolescentes, ciberacoso, convivencia escolar, Internet, dispositivos móviles, riesgos.

\section{Introduction and state of the question}

Social and institutional interest and concern about violent behaviour among school pupils has increased in recent years, as has its presence in the media ( $\mathrm{Sa}-$ huquillo, 2017). Both face-to-face bullying and the form which involves the use of ICT are subjects of analysis and concern at different levels, something which has led to a range of working definitions to describe these two phenomena.

While cyberbullying should be understood as an extension of the traditional form of bullying on virtual social networks and the Internet, some of the features of bullying - repetition, imbal-

ance of power, intent, and lack of justification - have to be redefined in the case of cyberbullying or online bullying. Repetition, for example, has been flagged as a problematic criterion, given that on occasions a single aggression using technological means can endure and perpetuate itself on the Internet, even though there was only a single action by the aggressor (Levy et al., 2012; Menesini et al., 2012; Slonje, Smith, \& Frisén, 2013). Similarly, factors such as the anonymity in which the bully can hide (Hinduja \& Patchin, 2008), the difficulty for the victim of stopping bullying on the Internet (Ovejero, Smith, \& Yubero, 2013), and the ease with which the audience can increase in the case of cyberbullying contribute to 
the victim's defencelessness and to harm being greater than in cases of traditional bullying (Estévez, Villardón, Calvete, $\mathrm{Pa}$ dilla, \& Orue, 2010).

Although bullying can occur in situations outside school -in public spaces such as parks or sports centres- and the online setting transcends schools, school is the location where most of children's social relationships occur, and so it is where bullying — traditional and online- is most common and most visible.

Among the elements relating to bullying - the family setting, the cognitive characteristics of bullies and victims, and social factors- it is important to mention ones that relate to the school setting, such as academic performance, peer pressure, lack of safety and supervision in schools, lack of measures to respond to diversity, the absence of a positive school climate, and lack of knowledge and limited control of the relationships students maintain on social networks, which, as Cook, Williams, Guerra, Kim, \& Sadek note (2010), are risk factors for cyberbullying.

There is no consensus when quantifying the prevalence of bullying and cyberbullying among school pupils in Spain. The data can, and indeed do, vary according to which tools are used to measure them, how the phenomenon is defined, and the age range considered, as Smith notes (2016). At a global level, in 2010 the WHO reported a prevalence of bullying at school which varied by country from $13 \%$ to $27 \%$ in Europe, with similar figures for 2016 (Currie et al., 2012; Inchley et al., 2016).
Regarding cyberbullying, Zych, Ortega-Ruiz, and Del Rey (2015) deduced from a review of over 60 studies that at least one in every five to seven minors are involved. At the European level, Livingstone, Haddon, Görzig, \& Ólafsson (2011) found that on average $6 \%$ of the population aged between 9 and 16 believed they had been victims of cyberbullying and $3 \%$ said they had been a bully on the Internet. In the case of Spain, Garmendia, Jiménez, Casado y Mascheroni (2016) established that among Internet users aged between 9 and 16 the prevalence of online victimisation was $12 \%$ and the prevalence of bullying was $8 \%$. Likewise, the survey carried out in 2016 by Save the Children is noteworthy thanks to the size of its sample, which included over 21,000 children and adolescents aged from 12 to 16 from Spanish public schools. This gave the result that $9.3 \%$ of those surveyed considered that they had suffered traditional bullying in the last two months, and $6.9 \%$ considered they had been victims of cyberbullying, with insults being the most frequent form of aggression (Save the Children, 2016).

Various channels are used for carrying out online aggression: harassment by telephone, recordings of physical attacks circulated by instant messaging or on sharing platforms, emails, spreading rumours and threats on social media, exclusion, etc. In this way, as the online habits of the school population and its access to different technological elements have increased and diversified, so too have forms of cyberbullying. It is worth noting that Internet use has now spread to virtually 
all of the school-age population; in Spain, on average, $95 \%$ of children and adolescents aged 10 to 15 use the Internet. The use of mobile phones, in turn, is strongly shaped by age: $25 \%$ of children have a mobile phone at the age of 10 ; practically half do at 11, and from 14 years of age, over $90 \%$ have a device for their own use (Spanish National Statistics Institute (INE), 2017). In Europe, $97 \%$ of the population aged between 15 and 24 has virtually daily access to the Internet, with $85 \%$ doing so from a smartphone (European Commission, 2015).

School is the key area for action on bullying among children and adolescents and the need for schools to intervene in the face of this phenomenon is upheld. However, school intervention on bullying currently faces complex challenges. For example, there are situations in which more than one school is involved in a single case of cyberbullying or, as it is deemed to have happened outside of school, responsibilities are watered down and nobody acts. Nonetheless, bullying causes harm to whole communities, and so it is necessary for all parties -family and society as well as the school - to be involved (Cohen-Almagor, 2018). Work to prevent different forms of violence among the student body as a whole is fundamental to prevent any form of bullying (Save the Children, 2016).

The field of psychology has suggested that the role of schools is as a force for cooperation between different parties such as the school and family as well as society and the community. Interventions must focus on both the aggressor and the victim and on the two of them jointly. Different types of intervention are recommended, both for conflict prevention and to improve coexistence in schools. These include primary intervention, to detect initial situations of mistreatment, and secondary intervention in the face of consolidated situations to provide therapeutic support or protection for victims, and monitor aggressors (Garaigordobil, 2011). Nonetheless, the best form of intervention proposed is to encourage harmonious coexistence in schools, stimulating social and emotional skills, cooperation, and conflict solving (Garaigordobil, 2015).

Del Rey, Estévez, and Ojeda (2018) refer to a variety of school programmes focussed on prevention or intervention in the case of cyberbullying. These programs focus on raising awareness in society and making students reflect, strengthening a critical attitude to the phenomenon and making them aware of security and the protection they should have on the Internet, and fostering good use of social media and the Internet (Del Rey, Estévez, \& Ojeda, 2018).

Evidence shows that, to varying degrees, cyberbullying complements bullying at school (Hinduja \& Patchin, 2009; Smith, Kwak, \& Toda, 2016). The aim of this work is to analyse the relationship between bullying and cyberbullying in Spain, starting from the basis that the two phenomena overlap, to describe the frequency with which they occur in different age ranges and with different levels of 
harm for their victims, and to explore the association between the roles of victim and aggressor in bullying processes, taking the school setting as the context.

\subsection{Material and methods}

This article analyses data obtained through a survey funded by the Spanish Ministry of the Economy and Companies, reference number CSO 2013-47304-R, which was carried out from April to June 2015. With regards to the population and sample, according to data from the Spanish National Statistics Institute, the reference population -children and adolescents aged between 9 and 16- was estimated to be $3,758,400$. Similarly, the Spanish National Statistics Institute, in the press release about ICT equipment and usage at home, estimated that among children and adolescents aged between 10 and 15, use of the Internet is universal. Consequently, with a margin of error of $4.45 \%$ and a confidence level of $95.5 \%$, it was decided to use a sample of 500 children and adolescents. The fieldwork involved surveying children and adolescents aged between 9 and 16, all of whom were Internet users, and their parents. In each family home, the parent most involved with the online activity of the child or adolescent was interviewed. In order to maximise the quality of the responses, the questionnaires were completed in the homes of the families and self-administered questionnaires were also used for the more sensitive questions asked to the children and adolescents.

The sample was stratified by region and level of urbanisation and sampling points were selected using the census sections. After this, the addresses of the homes were selected at random using the random route process. The survey analysed Internet access and usage, the online activities of the children and adolescents, the incidence of the risks and the subjective perception of the harm caused by them, as well as communicative practices, digital skills, aspects relating to excessive use, and parental mediation.

In this article we analyse the results relating to bullying between peers considering the medium through which it occurs -online and/or offline - and the roles of the children and adolescents involved in the incidents of violence. The statistical analysis will fundamentally be descriptive as the relatively small number of children and adolescents who are victims and/or aggressors does not allow for more elaborate statistical analyses. The frequency analyses will be structured by the age of the children and adolescents as this has a direct influence on the development of digital skills, personality maturation, and possessing mobile phones. The prevalence of bullying will be analysed according to its different forms, the frequency of the roles involved for bullying and cyberbullying, the association between the two roles, and the relationship between harm and age according to type of bullying.

\section{Analysis and results}

2.1. Context and forms of cyberbullying

Table 1 shows how the incidence of face-to-face bullying is much higher than that for cyberbullying in any of its various forms. 
TABLE 1. Percentages for forms in which children and adolescents have suffered bullying in the last 12 months by age (absolute frequencies in parentheses).

\begin{tabular}{|c|c|c|c|c|c|}
\hline \multirow{2}{*}{$\%$} & \multicolumn{4}{|c|}{ Age } & \multirow{2}{*}{ Total } \\
\hline & $9-10$ & $11-12$ & $13-14$ & $15-16$ & \\
\hline In person, face-to-face & $27(36)$ & $27(34)$ & $27(34)$ & $20(23)$ & $25(127)$ \\
\hline Mobile phone calls & 0 & $1(1)$ & $2(2)$ & $3(3)$ & $1(6)$ \\
\hline $\begin{array}{l}\text { Through messages on my phone (SMS } \\
\text { or MMS) }\end{array}$ & 0 & $1(1)$ & $2(3)$ & $3(3)$ & $1(7)$ \\
\hline $\begin{array}{l}\text { On a social network (Tuenti, Facebook, } \\
\text { etc.) }\end{array}$ & $1(1)$ & $4(5)$ & $6(7)$ & $8(9)$ & $4(22)$ \\
\hline $\begin{array}{l}\text { On a platform (YouTube, Instagram, } \\
\text { Flickr) }\end{array}$ & $2(3)$ & $3(4)$ & $6(7)$ & $2(2)$ & $3(16)$ \\
\hline $\begin{array}{l}\text { By instant messaging (MSN, WhatsApp, } \\
\text { Skype) }\end{array}$ & $2(2)$ & $4(5)$ & $7(9)$ & $9(10)$ & $5(26)$ \\
\hline In a chatroom & $3(4)$ & $6(7)$ & $4(5)$ & $2(2)$ & $4(18)$ \\
\hline $\begin{array}{l}\text { Any form of bullying through the Inter- } \\
\text { net or mobile devices }\end{array}$ & $8(10)$ & $13(16)$ & $14(17)$ & $15(17)$ & $12(60)$ \\
\hline TOTAL victims & $33(43)$ & $32(40)$ & $33(40)$ & $28(31)$ & $32(154)$ \\
\hline Non-victims & $67(91)$ & $68(87)$ & $67(91)$ & $72(84)$ & $68(346)$ \\
\hline
\end{tabular}

Q33: If someone treated you like this, how did it happen? (Please mark as many boxes as necessary.)

Sample: all children and adolescents who use the Internet $(\mathrm{N}=500)$.

Source: Own elaboration.

Twelve per cent of the children and adolescents reported having been victims of cyberbullying, although the percentage of victims of face-to-face bullying (25\%) is over twice the rate for victims involving telephones or the Internet. The data corresponding to the prevalence of each type show that the various types overlap. So, the sum of the relative weight of the victims of the various online forms is higher than the total percentage of online victims $(12 \%)$. Likewise, the percentage for face-to-face victims $(25 \%)$ plus the percentage for online victims (12\%) is higher than the total percentage for victims of bullying (32\%). This shows that the types of bullying overlap. For example, a particular child might be bullied faceto-face and by instant messaging and/ or in a chatroom simultaneously. In any case, the data show that cyberbullying is not the dominant model of aggression between peers. Instead, the data seem to show the contrary; face-to-face bullying is predominant with other forms added to it.

The most frequent channels through which cyberbullying occurs are instant messaging (WhatsApp, 5\%), social networks (4\%), and chatrooms (4\%). This last category is mainly used by preadolescents. In contrast, among those aged over 13-14, online bullying mainly occurs through instant messaging (7-9\%) and on social media (6-8\%), while in the 13-14 age group, victimisation on sharing platforms is most noticeable $(6 \%)$. 
2.2. Victims and aggressors by age and type of bullying

When the term bullying is used, it generally refers to the victimisation to which children and adolescents are subjected. Nonetheless, in this work we will analyse both types of violent behaviour, differentiating the two main roles of the people involved - victim and bully- depending on the two principal types of bullying.

The difference between the various age groups is striking: for those aged under 15 , offline bullying clearly predominates, with around $27 \%$ in the 9 to 14 band compared with $20 \%$ in the 15 to 16 band. In contrast, the prevalence of victimisation through cyberbullying tends to increase with age, in particular from the age of 11 , reaching $15 \%$ in the 15 to 16 age group. In this age group, the difference between the two types of bullying is $5 \%$, while in the younger age groups, it is much higher: between the ages of 11 and 14 the relative frequency of offline victimisation is double that of online victimisation and in the youngest age group it is three times higher.

In general, the prevalence of bullying -offline and online - is slightly lower than that of victimisation; however, the difference between the frequencies of the two roles is smaller in the case of cyberbullying. So much so that among young people aged 13 and 14, both frequencies are exactly the same; in this age range there are as many victims as bullies involved in cyberbullying incidents. The evidence shows that technology facilitates insults, revenge, and switching of roles between the agents involved, as was also established in the interviews and discussion groups held in previous pieces of research.

GRAPH 1. Victimization by age: bullying v. cyberbullying.

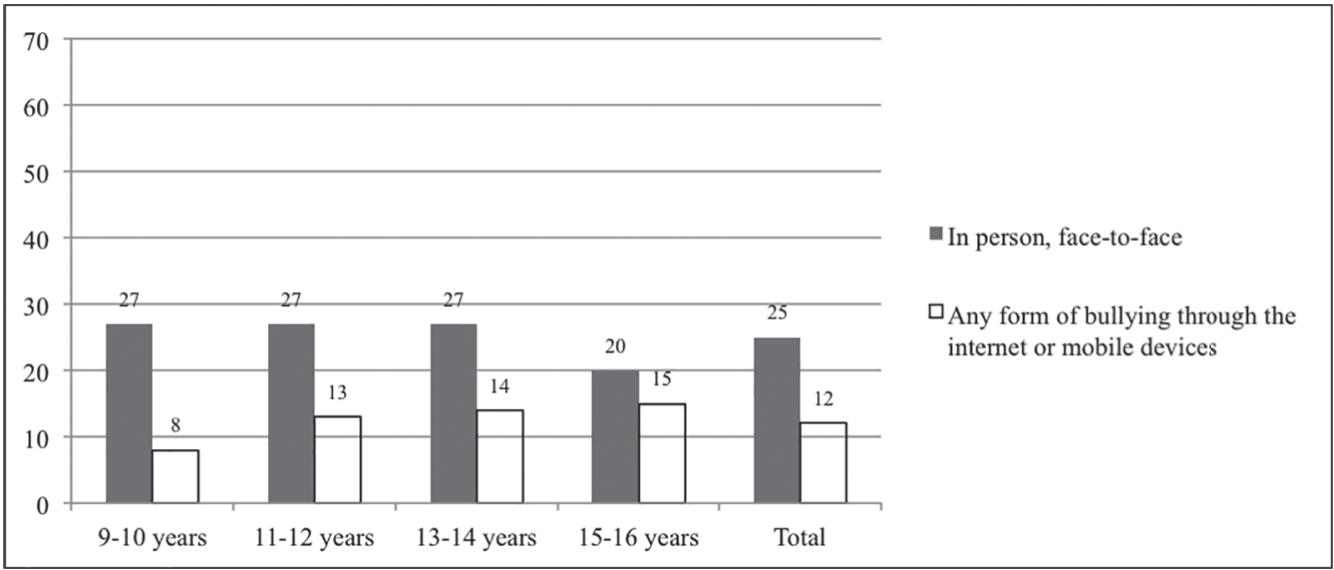

Q33: If someone treated you like this, how did it happen? (Please mark as many boxes as necessary.)

Sample: all children and adolescents who use the Internet $(\mathrm{n}=500)$.

Source: Own elaboration. 
In general, the percentage of bullies tends to increase with age up to 13-14 where it reaches $23 \%$ in the offline form and $14 \%$ online, while in the $15-16$ age band it falls to $16 \%$ and $8 \%$ respectively. Both types of bullying -online and offline- are most prevalent in the 13-14 age group.

GRAPH 2. Victimization by age: bullying v. cyberbullying.

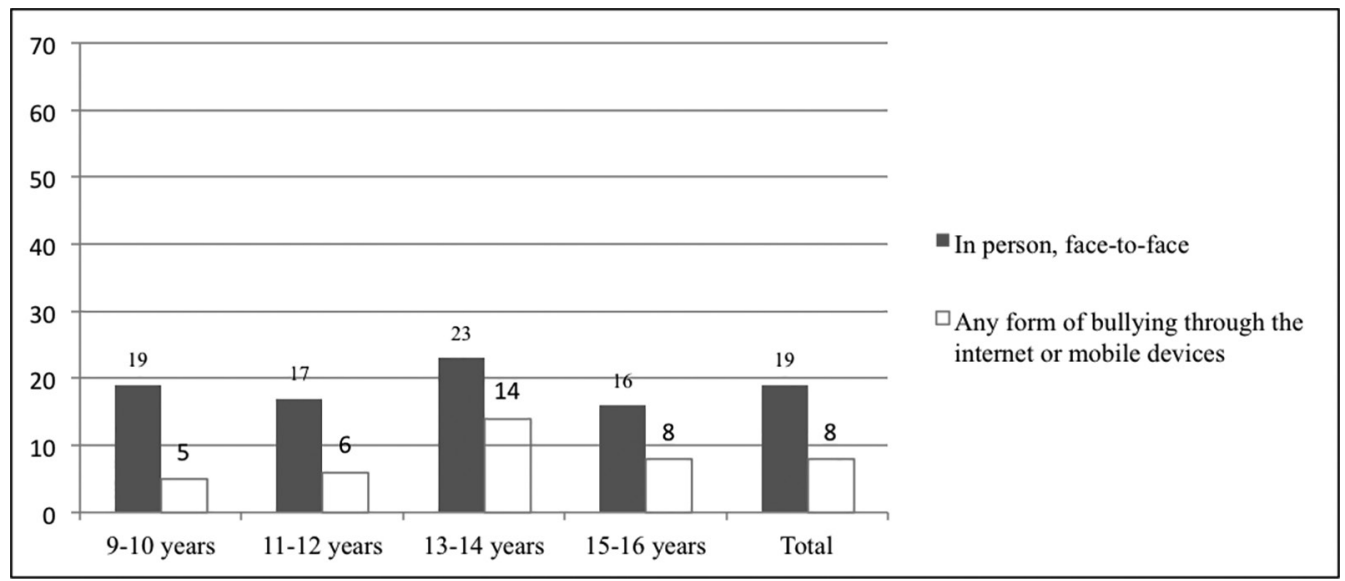

Q34: In the last 12 months, have you treated somebody else like this? If so, how did you do it? (Please mark as many boxes as necessary.)

Sample: all children and adolescents who use the Internet $(\mathrm{N}=500)$.

Source: Own elaboration.

\subsection{Relationship between aggressor} and victim in the online setting

Table 2 shows that there is a significant association (Chi squared $=138.5$, significance $=0.000$ ) between the roles of bully and victim in the online setting. In the total percentages the relatively low incidence of children and adolescents involved in incidents of cyberbullying tends to blur the trends. Nonetheless, participation in episodes of cyberbullying has an enormous impact on the likelihood of becoming a victim of bullying. If, on average, $12 \%$ of minors have been victimised, this datum varies considerably depending on whether they have been involved in incidents of cyberbullying as the bully. So, among those who have not bullied other children and adolescents online, the per- centage who say they have been victims of bullying is relatively low (7\%), while among the children and adolescents who have bullied people, three out of every four have also been victims of online bullying.

TABLE 2. Association between the roles of bully and victim in cyberbullying.

\begin{tabular}{|l|c|c|c|}
\hline \multirow{2}{*}{$\begin{array}{c}\text { \% Victims } \\
\text { online }\end{array}$} & \multicolumn{2}{|c|}{ \% Aggressors } & \multirow{2}{*}{ Total } \\
\cline { 2 - 3 } Not victims & $86(428)$ & $2(12)$ & $88(440)$ \\
\hline Victim & $6(32)$ & $6(28)$ & $12(60)$ \\
\hline Total & $92(460)$ & $8(40)$ & $100(500)$ \\
\hline
\end{tabular}

Q34: In the last 12 months, have you treated somebody else like this? If so, how did you do it? Online.

Q33: If someone treated you like this, how did it happen? Online.

Sample: all children and adolescents who use the Internet $(\mathrm{n}=500)$.

Source: Own elaboration. 


\subsection{Association between harm and age by type of bullying}

Another aspect to take into account when analysing bullying and cyberbullying is the harm they cause to the victims. Previous research has found that bullying —online and offline- is in general the most damaging experience of risk (Livingstone et al., 2011; Mascheroni \& Cuman, 2014) compared with other potential risks such as accessing unsuitable content or contact with strangers.

Among children and adolescents who have been victims of face-to-face bullying, on average $40 \%$ said they felt very upset, a slightly lower percentage said they felt a bit upset (38.6\%), and $21 \%$ said they did not feel upset. The results show that the experience of harm is strongly shaped by age. Accordingly, the proportion who felt very upset increases to $47 \%$ in the 11 12 age group. In the 13-14 age group it falls to $41 \%$, and in the $15-16$ age group it drops notably to $22 \%$. The proportion of those who said they had felt a bit upset is slightly lower than those who felt very upset between the ages of 9 and 14 and in the 15-16 age group it increases because the relative weight of those who felt very upset reduces drastically. Finally, the proportion of people who were not upset is around $14 \%$ between the ages of 9 and 12. It increases by almost 10 percentage points in the 13-14 age group, and is $39 \%$ in the 15-16 age group. The data show that from the age of 13, the development of resilience and digital skills as well as personality maturation can contribute to young people managing situations of conflict more effectively and being less vulnerable to the harm caused by face-toface bullying.

The results show that on average the proportion of children and adolescents who said they had felt very upset because of an episode of cyberbullying (45\%) is greater than the percentage who said they felt that way because of an episode of face-to-face bullying (40\%). In contrast, the percentage of children and adolescents who said they had felt a bit upset is lower in the case of cyberbullying (28\% compared with $39 \%$ ). The data show that the subjective perception of harm caused by cyberbullying is more polarised: people who report feeling very upset are relatively more numerous, but the ones who report not feeling upset are also more numerous.

Regarding age, it is worth noting that among under $12 \mathrm{~s}$, the percentage who felt very upset is between $40 \%$ and $44 \%$ compared with $30 \%$ who say cyberbullying did not affect them. This last datum could indicate that at this early age, somewhat under one in three children is already developing resilience. Nonetheless, the data for the 13-14 age group entirely contradict this. This group's vulnerability to online victimisation is very notable: $65 \%$ state that they felt very upset, $24 \%$ a bit upset, and just $12 \%$ were not upset. This age group's very high vulnerability to cyberbullying could possibly be associated with the importance these young people place on their online reputation, which would be seriously affected by incidents of online violence. In contrast, among 
those aged 15 and 16 , the percentage who felt very upset falls notably ( $29 \%)$, as also happens in the case of bullying, while the same percentage felt a bit upset and not upset $(35 \%)$.

GRAPH 3. Level of harm among victims of face-to-face bullying.

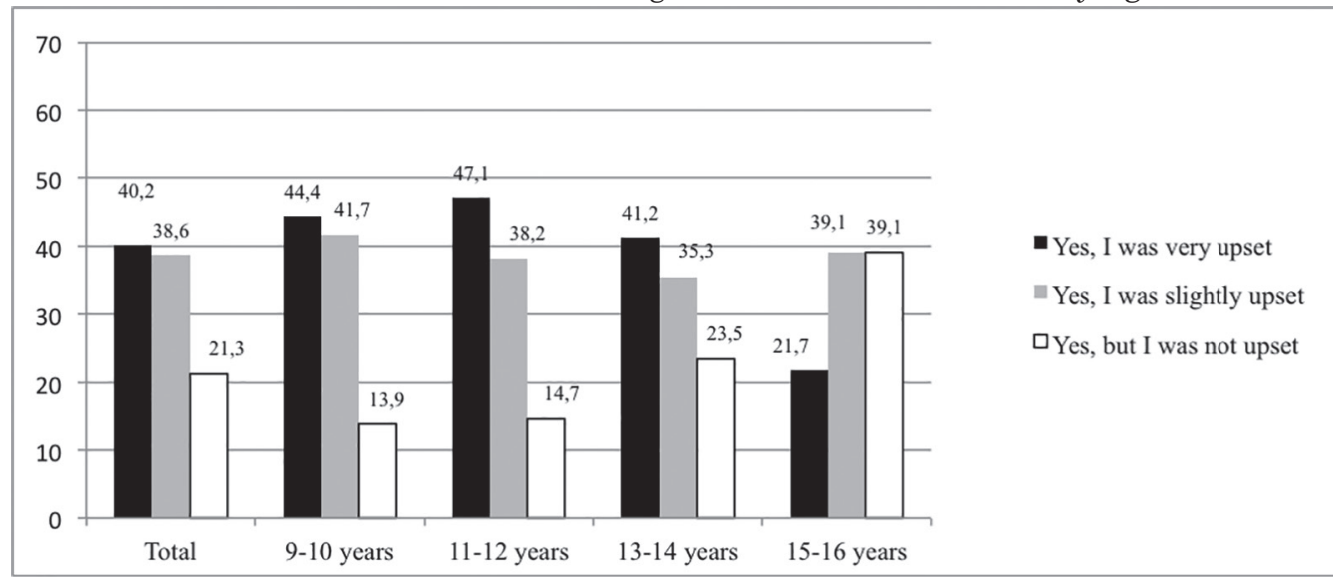

Q32: Has anyone treated you like this in the last 12 months? If so, how upset were you? Sample: all children and adolescents who use the Internet and have been victims of face-toface bullying $(n=127)$.

Source: Own elaboration.

GRAPH 4. Level of harm among victims of cyberbullying.

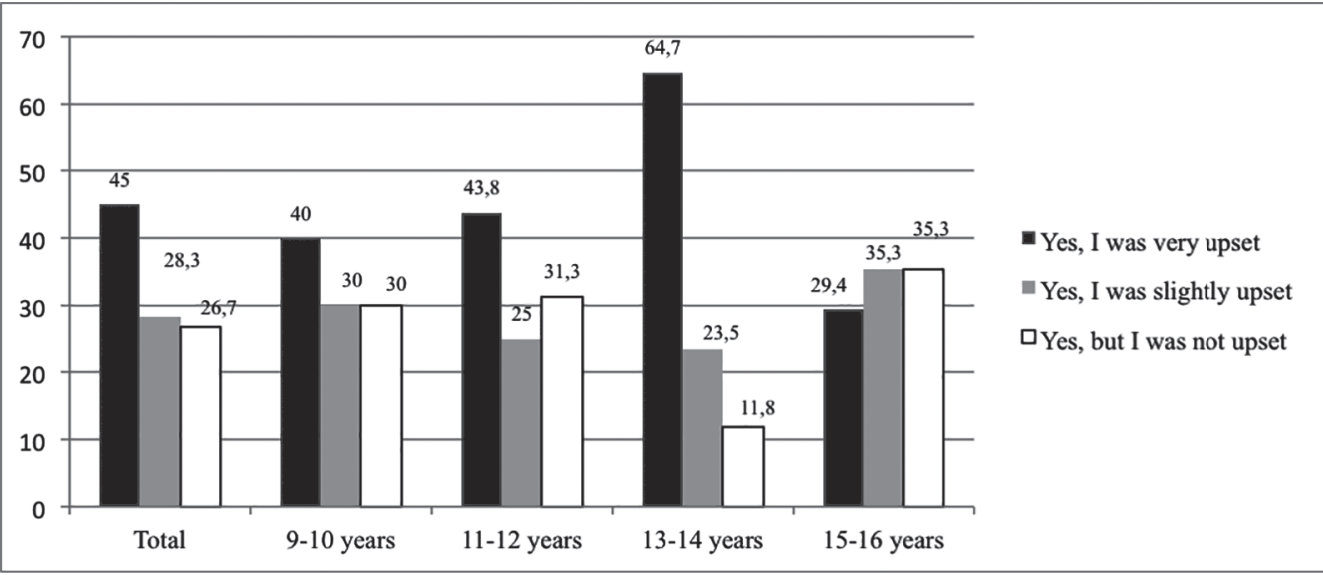

Q32: Has anyone treated you like this in the last 12 months? If so, how upset were you?

Sample: all children and adolescents who use the Internet and have been victims of cyberbullying $(\mathrm{n}=60)$.

Source: Own elaboration. 


\section{Discussion}

The evidence shows that situations of online bullying take place in a setting, in which there are many more cases of face-to-face bullying. Online bullying, or cyberbullying is a form of bullying, which is superimposed on other already-existing types, something which agrees with what previous research and reviews have shown (Ybarra, Boyd, Korchmaros, \& Oppenheim, 2012; Zych et al., 2015; Garmendia et al., 2016). Both types of bullying display an increasing trend, and so the prevalence of bullying in general —online and offline- has doubled compared to the year 2010: $15 \%$ of children and adolescents aged between 9 and 16 (Garmendia, Garitaonandia, Martínez, \& Casado, 2011) compared with $32 \%$ in 2015 . This notable increase in the incidence of bullying could relate to a greater ability to identify situations of abuse and bullying by people who suffer from it and to greater social awareness of it (Garmendia et al., 2018).

Regarding the relationship between the prevalence of cyberbullying and age, while Del Rey et al. (2018) found mixed results, in our work, the differences by age group show a progression of violent online behaviour between peers: the frequency of cyberbullying increases with age unlike with face-to-face bullying (Garmendia, Garitaonandia, Martínez, \& Casado-Calatayud, 2018; Barboza, 2015; Cappadocia, Craig, \& Peppler, 2013; Kowalski \& Limber, 2007). The number of people who suffer from cyberbullying increases as their age increases, although this bullying behaviour is carried out by fewer people. Therefore, a smaller number of aggressors bully and victimise a larger number of students in the later years of compulsory secondary education. However, in offline bullying, the frequency increases with age but from the age of 15 there is a change in trend, with face-to-face bullying falling.

The high prevalence of bullying behaviour in the 13-14 age group is striking. This coincides with the first stage of secondary education, where supervision of pupils in schools is relatively limited while at the same time the possession of mobile phones also increases notably (INE, 2017), something often regarded as a rite of passage as young people often receive a smartphone as a present to mark their move to secondary education (Mascheroni \& Cuman, 2014). In contrast, from the age of 15-16, the prevalence of face-to-face bullying falls notably, something that can be interpreted as part of the process of maturing and learning about managing emotions and the consequences of actions.

In this sense, it is important to consider that anonymity and ease of use and immediacy contribute to online insults being seen easy and free from consequences. This evidence can therefore be interpreted as a trivialisation of online violence. On many occasions, the context of the aggression is connected to young couples where, in an immature handling of a break-up, one of them distributes messages or images of the former-partner as blackmail or revenge to damage their reputation (Roca, 2015). This is consistent with the association Görzig proposes (2011) between cyberbullying, sensation seeking, and the difficulties associated with maturation. As 
a conclusion in this regard, and in view of the development of cyberbullying by age group, it can be deduced that paying special attention to the first stage of secondary education, where the near-universal penetration of smartphones coincides with a lack of skill in managing social relationships, is vital for preventing and tackling situations of online bullying. On these lines, Garaigordobil claims that "if there is no preventive intervention, cyberbullying can not only not be expected to fall but instead can be expected to increase in the 12 to 18 age group" (2015, p. 1074), noting that good habits and prevention plans for cyberbullying are fundamental for learning to relate online.

There is a significant association online between the roles of bully and victim. The data do not allow us to clarify what the sequence is in the interplay between the two roles, if victimisation promotes bullying behaviour or if bullying behaviour leads to subsequent victimisation of the bullies. Nonetheless, there is ample evidence from previous research to support this connection, such as the work by Görzig (2011), which showed that around $60 \%$ of bullies had also been bullied, and the work by Lampert and Donoso, who also stated that "being a cyberbully is the best predictor of being a victim of cyberbullying" and noted that the boundaries between roles are harder to trace in cyberbullying than in face-to-face bullying (2012, p. 146).

Regarding the incidence of harm, understood as a subjective experience which can vary with each experience of bullying, the data show that the subjective percep- tion of the harm caused by cyberbullying is more polarised than in the case of faceto-face bullying: people who report being very upset are relatively more numerous, but people who say they were not affected by it are also more numerous. Strong shaping by age is also observed: as the age of the victims increases, the proportion of severe harm falls, but this differs somewhat between face-to-face and online bullying. From the age of 13, the development of resilience and maturation can contribute to young people managing conflicts more effectively and being less vulnerable to the harm caused by conventional bullying. And yet vulnerability to cyberbullying is extraordinary in the 13-14 age range: $65 \%$ of those affected said they felt very upset, $24 \%$ somewhat upset, and just $12 \%$ did not feel upset. As noted above, this very high level of vulnerability is probably connected to the importance of the online reputation in this age group, and with the perception that aggression might have a universal reach (Ruiz, Martín, López, \& Hernán, 2016), which has an impact on the particular interest in conflict prevention and management tasks among these children and adolescents.

\section{Conclusions}

The close association identified in our work between the roles of bully and victim indicate that the school climate is affected by situations of violence, which arise in turn are fed by episodes of aggression, and in which those children who observe, suffer and/or reproduce it also participate. All of this means that school intervention is necessary to protect victims and re-edu- 
cate aggressors (Save the Children, 2016), and it is also an important unifying element which is independent of each family's starting regarding, for example, digital skills (Garmendia et al., 2016). Acting in schools involves difficulties such as the school law of silence which leads to teachers being unable to help victims because they are not always aware of what is happening (Del Rey et al., 2018), or lack of specific training to handle this sort of situation. In this sense, it is important to detect situations of bullying, and also to have action protocols and prevention plans, taking as reference points elements that have been proven to be effective against traditional bullying (Ttofi \& Farrington, 2011), as well as other specific ones given the differences detected between the two types of aggression.

The ombudsman has recommended that educational centres have increasing intervention on bullying, working with families and counsellors, and providing students with interpersonal relationship skills and communication strategies. In other words, solving issues with coexistence in a given school, without relying so much on external interventions, social services, the police, and courts (Defensor del Pueblo, 2007). In this respect, there is evidence for a reduction in the number of problems between students in schools with a plan for education in problem solving (Caballero, 2010).

But reality differs greatly from what was suggested. The cuts resulting from the financial crisis have meant that many schools can no longer have a PTSC (Spe- cialist Community Service Teacher), a figure supporting harmonious coexistence who in theory would be the person responsible for implementing protocols or plans against bullying in schools, something which, in light of the evidence shown here, is a grave error. Consequently, it is noteworthy that "in the Spanish Law to Improve the Quality of Education's mentions of teacher training and granting resources to schools, educational quality is constantly linked to curriculum subjects, and prevention of violence is not regarded as an essential part of the desired educational quality" (Del Rey et al., 2018, p. 86). At the same time, there is still little regulatory support for tackling violence in schools and what there is only partially matches the suggestions by the ombudsman and by leading researchers on the topic in our setting, and the creation of specific legislation relating to school violence continues to be vital (Cerezo \& Rubio, 2017). Overall, the contrast between the growing social interest in mistreatment between peers and the lack of political commitment to preventing it is worrying.

In this sense, the report "Los derechos de los niños y niñas en el sistema educativo en España" "The rights of children in the Spanish educational system", Larrañaga, 2016) underlines the social acceptability of violence in some school settings, where aggressors tend to replicate patterns of violence in relationships with their peers without being aware of the harm caused to the victim. It is apparent that the school setting is very prone to permeation by violence from other settings in current society, which is characterised by individual- 
ism, competitiveness, and a fast pace of life where interpersonal relationships are becoming increasingly conflictive. Recreating harmonious coexistence in schools is a challenge which should involve all agents - teachers, students, and parents - to transform the culture of mistreatment into a culture of good treatment.

It has also been shown that a friendly and supportive climate in schools has positive effects on students and reduces the likelihood of bullying, both online and face-to-face. Research shows that when young people feel connected to their schools, in an impartial, pleasant, and positive climate of trust, acknowledged involvement in all types of bullying - physical, verbal, or online - is lower (Cohen-Almagor, 2018; Cohen, Twemlow, Berkowitz, \& Comer, 2015; Williams \& Guerra, 2007). discussing the problem in the school and trying to explain the effects of bullying on victims. But the work of schools must take place alongside the parents to raise awareness and control the problem. Prevention programmes in schools, which offer support for students, are necessary, but parental intervention is also required (Cohen-Almagor, 2018).

And schools cannot tackle this social problem alone. The latest research into the topic suggests it is necessary to implement measures in the face of the social ills created by the Internet, and it calls for responsible cooperation between parents, schools, governments and institutions, non-governmental organisations (NGOs), and the people in charge of social networks. Intimidation is a problem which affects and concerns us all, and so it must be approached in an interdisciplinary manner. Responsibility and accountability must be shared by all parties: parents, teachers, schools, NGOs and businesses, and must encompass different countries and the international community $\mathrm{C}_{0}$ hen-Almagor, 2018).

\section{References}

Barboza, G. E. (2015). The association between school exclusion, delinquency and subtypes of cyber - and F2F - victimizations: Identifying and predicting risk profiles and subtypes using latent class analysis. Child Abuse \& $\mathrm{Ne}$ glect, 39, 109-122. doi: https://doi.org/10.1016/j. chiabu.2014.08.007

Caballero, M. J. (2010). Un estudio sobre buenas prácticas. Revista paz y conflictos, 3, 154-169.

Cappadocia, M. C., Craig, W. M., \& Pepler, D. (2013). Cyberbullying prevalence, stability, and risk factors during adolescence. Canadian Journal of School Psychology, 28 (2), 171-192. doi: https://doi.org/10.1177/0829573513491212

Casas, J. A., Del Rey, R., \& Ortega-Ruiz, R. (2013). Bullying and cyberbullying: convergent and divergent predictor variables. Computers in $\mathrm{Hu}$ man Behaviour, 29 (3), 580-587. doi: https://doi. org/10.1016/j.chb.2012.11.015

Cerezo, F., \& Rubio, F. J. (2017). Medidas relativas al acoso escolar y ciberacoso en la normativa autonómica española. Un estudio comparativo. Revista Electrónica Interuniversitaria de Formación del Profesorado, 20 (1), 113-126.

Cohen, J., Espelage, D. L., Twemlow, S. W., Berkowitz, M. W., \& Comer, J. P. (2015). Rethinking effective bully and violence prevention efforts: Promoting healthy school climates, positive youth development, and preventive bully-victim-bystander behavior. International Journal of Violence \& Schools, 15 (1), 2-40. 
Cohen-Almagor, R. (2018). Social responsibility on the Internet: Addressing the challenge of cyberbullying. Aggression and Violent Behavior, 39, 42-52. doi: https://doi.org/10.1016/j. avb.2018.01.001

Cook, C. R., Williams, K. R., Guerra, N. G., Kim, T. E., \& Sadek, S. (2010). Predictors of bullying and victimization in childhood and adolescence: a meta-analytic investigation. School Psychology Quarterly, 25 (2), 65-83. doi: http:// dx.doi.org/10.1037/a0020149

Currie, C., Zanotti, C., Morgan, A., Currie, D., De Looze, M., \& Roberts, C. (2012). Health policy for children and adolescents (Report n.6). Social determinants of health and well-being among young people: Health Behaviour in School-aged Children (HBSC) study: International report from the 2009/2010 survey. Copenhage: World Health Organization, Regional Office for Europe.

Defensor del Pueblo (2007). Violencia escolar: el maltrato entre iguales en la educación secundaria obligatoria 1999-2006. Madrid: Defensor del Pueblo.

Del Rey, R., Estévez, M., \& Ojeda, M. (2018). El ciberbullying y su respuesta educativa. En E. Jiménez, M. Garmendia y M. A. Casado, Entre selfies y whatsapps. Oportunidades y riesgos para la infancia y la adolescencia conectada (pp. 125-139). Barcelona: Gedisa.

Del Rey, R., Elipe, P., \& Ortega-Ruiz, R. (2012). Bullying and cyberbullying: overlapping and predictive value of the co-occurrence. Psicothema, 24 (4), 608-613.

Estévez, A., Villardón, L., Calvete, E., Padilla P., \& Orue, I. (2010). Adolescentes víctimas de cyberbullying: prevalencia y características. Behavioral Psychology/Psicología Conductual, 18 (1), 73-89.

European Commission (2015). Special Eurobarometer 423: Cyber Security. Bruselas: European Union.

Garaigordobil, M. (2011). Prevalencia y consecuencias del cyberbullying: una revisión. International Journal of Psychology and Psychological Therapy, 11 (2), 233-254.

Garaigordobil, M. (2015). Ciberbullying en adolescentes y jóvenes del País Vasco: Cambios con la edad. Anales de psicología, 31 (3), 1069-1076. doi: https://doi.org/10.6018/ analesps.31.3.179151
Garmendia, M., Garitaonandia, C., Martínez, G., \& Casado, M. A. (2011). Riesgos y seguridad en Internet: Los menores españoles en el contexto europeo. Bilbao: EU Kids Online, Universidad del País Vasco / Euskal Herriko Unibertsitatea. Retrieved from http://www.lse.ac.uk/media@lse/ research/EUKidsOnline/EU\%20Kids\%20II\%20 (2009-11)/National\%20reports/Spanish\%20 report.pdf (Consulted on 29/10/2018).

Garmendia, M. Jiménez, E., Casado, M. A., \& Mascheroni, G. (2016). Net Children Go Mobile: Riesgos y oportunidades en Internet $y$ el uso de dispositivos móviles entre menores españoles (2010-2015). Madrid: Red.es / Universidad del País Vasco / Euskal Herriko Unibertsitatea. Recuperado de https://addi.ehu.es/ handle/10810/21546 (Consulted on 29/10/2018).

Garmendia, M., Casado, M. A., Jiménez, E., \& Garitaonandia, C. (2018). Oportunidades, riesgos, daño y habilidades digitales de los menores españoles. In E. Jiménez, M. Garmendia, \& M. A. Casado, Entre selfies y whatsapps. Oportunidades y riesgos para la infancia y la adolescencia conectada (pp. 31-54). Barcelona: Gedisa.

González-Calatayud, V. (2018). Victims of cyberbullying in the Region of Murcia: a growing reality. Journal of New Approaches in Educational Research, 7 (1), 10-16.

Görzig, A. (2011). Who bullies and who is bullied Online?: a study of 9-16 year old Internet users in 25 European countries. London: EU Kids Online network. Retrieved from http://eprints. lse.ac.uk/39601 (Consulted on 29/10/2018).

Hinduja, S., \& Patchin, J. W. (2008). Cyberbullying: an exploratory analysis of factors related to offending and victimization. Deviant Behavior, 29 (2), 129-156. doi: https://doi. org $/ 10.1080 / 01639620701457816$

Hinduja, S., \& Patchin, J. W. (2009). Bullying beyond the schoolyard: Preventing and responding cyberbullying. Thousand Oaks, CA: Sage Publications.

Inchley, J., Currie, D., Young, T., Oddrun, S., \& Torbjorn, T. (2016). Health policy for children and adolescents, no. 7. Growing up unequal: gender and socioeconomic differences in young people's health and well-being. Health Behavior in Schoolaged Children (HSB) Study: International report from the 2013/2014 survey. Copenhagen: World Health Organization, Regional Office for Europe. 
Instituto Vasco de Evaluación e Investigación Educativa (ISEI-IVEI) (2017). Maltrato entre iguales en Euskadi 2016. Bilbao: Basque Government. Education Deparment. Retrieved from https://bit.ly/2U7Ivhp (Consulted on 09/04/2019).

Kowalski, R. M., \& Limber, S. P. (2007). Electronic Bullying Among Middle School Students. Journal of Adolescent Health, 41 (6), 22-30. doi: https://doi.org/10.1016/j.jadohealth.2007.08.017

Lampert, C., \& Donoso, V. (2012). Bullying. In S. Livingstone, L. Haddon, \& A. Görzig (Eds.), Children risk and safety on the Internet (pp. 139-148). Bristol: Policy Press.

Larrañaga, K. P. (2016). Los derechos de los niños y niñas en el sistema educativo en España. Análisis de la situación. Barcelona: Asociación GSIA / Fundación Educo. Retrieved from http://www. bienestaryproteccioninfantil.es/fuentes1.asp $?$ sec $=10 \&$ subs $=25 \& \operatorname{cod}=3460 \&$ page $=\& v=2$ (Consulted on 29/10/2018).

Livingstone, S., Haddon, L., Görzig, A., \& Ólafsson, K. (2011) Risk and safety on the Internet. The perspective of European children. Full Findings. London: EU Kids Online, The London School of Economics and Political Science (LSE).

Levy, N., Cortesi, S., Crowley, E., Beaton, M., Casey, J., \& Nolan, C. (2012). Bullying in a networked era: A literature review. Berkman Center Research Publication, 201217. Recuperado de http://cyber.law.harvard. edu/publications/2012/kbw_bulling_in_a_ networked_era (Consulted on $\overline{2} 9 / 10 / 2018$ ).

López-Pradas, I. C., Romera, E. M., Casas, J. A., \& Ortega-Ruiz, R. (2017). Cybergossip and cyberbullying during primary school years. $P$ sicología Educativa, 23 (2), 73-80. doi: https:/doi. org/10.1016/j.pse.2017.05.007

Mascheroni, G., \& Cuman, A. (2014). Net Children Go Mobile: Final report. Deliverables D6.4\&D5.2. Milano: Educatt.

Menesini, E., Nocentini, A., Palladino, B. E., Frisén, A., Berne, S., Ortega-Ruiz, R., ... Smith, P. K. (2012). Cyberbullying definition among adolescents: a comparison across six European countries. Cyberpsychology, behavior, and social networking, 15 (9), 455-463. doi: https:// doi.org/10.1089/cyber.2012.0040
Ortega, R., Calmaestra, J., \& Mora, J. (2008). “Cyberbullying". International Journal of Psychology and Psychological Therapy, 8 (2) 183-192.

Ovejero, A., Smith, P. K., \& Yubero, S. (Coords.) (2013). El acoso escolar y su prevención: perspectivas internacionales. Madrid: Biblioteca Nueva.

Roca, G. (2015). Las nuevas tecnologías en niños y adolescentes. Guía para educar saludablemente en una sociedad digital. Barcelona: Hospital Sant Joan de Déu.

Rubio Sáiz, M. (2013). Estudio sobre la percepción del profesorado en educación secundaria obligatoria del acoso escolar. Revista de Educación Social, 16. Retrieved from http://www.eduso. net/res/pdf/16/acos_res_\%2016.pdf (Consulted on 09/04/2019).

Ruiz Benítez, B., Martín Barato, A., López Catalán, B., \& Hernán García, M. (2016). ¿ Convivencia o Bullying?: análisis, prevención y afrontamiento del acoso entre iguales. Granada: Consejería de Salud. Escuela Andaluza de Salud Pública.

Sahuquillo Verdet, F. (2017). Fuentes y encuadres del discurso mediático del acoso escolar en los periódicos El Mundo y El País. Doxa Comunicación, 25, 169-192.

Save the Children (2016). Yo a eso no juego. Bullying y cyberbullying en la infancia. Recuperado de https://www.savethechildren.es/sites/ default/files/imce/docs/yo_a_eso_no_juego.pdf (Consulted on 29/10/2018).

Slonje, R., Smith, P. K., \& Frisén, A. (2013). The nature of cyberbullying, and strategies for prevention. Computers in Human Behavior, 29 (1), 26-32. doi: https://doi.org/10.1016/j. chb.2012.05.024

Smith, P. K. (2016). Bullying escolar. In S. Yubero, E. Larrañaga, R. Navarro (Eds.), La violencia en las relaciones humanas: contextos y entornos protectores del menor (pp. 9-29). Cuenca: Ediciones de la Universidad de Castilla-La Mancha.

Smith, P. K., Kwak, K., \& Toda, Y. (2016). School bullying in different cultures. Cambridge: Cambridge University Press.

Spanish National Statistics Institute (INE) (2017). Encuesta sobre Equipamiento y Uso de Tecnologías de Información y Comunicación en los Hogares 2017. Madrid: Instituto Nacional de Estadística (Spanish National Statistics Institute). 
Bullying and cyberbullying: victimisation, harassment, and harm. The need to intervene in the...

Ttofi, M. M., \& Farrington. D. P. (2011). Effectiveness of school-based programs to reduce bullying: a systematic and meta-analytic review. Journal of Experimental Criminology, 7 (1). 27-56. doi: https://doi.org/10.1007/s11292-0109109-1

Williams, K. R., \& Guerra, N. G. (2007). Prevalence and predictors of Internet bullying. The Journal of Adolescent Health, 41 (6), S14-S21.

Ybarra, M. L., Boyd, D., Korchmaros, J. D., \& Oppenheim, J. (2012). Defining and measuring cyberbullying within the larger context of bullying victimization. Journal of Adolescent Health, 51 (1), 53-58. doi: https://doi.org/10.1016/j. jadohealth.2011.12.031

Zych, I., Ortega-Ruiz, R., \& del Rey, R. (2015). Systematic review of theoretical studies on bullying and cyberbullying: facts, knowledge, prevention, and intervention. Aggression and Violent Behaviour, 23, 1-21. doi: https://doi. org/10.1016/j.avb.2015.10.001

\section{Authors' biographies}

Maialen Garmendia Larrañaga is a $\mathrm{PhD}$ in Sociology and Political Sciences from the Universidad de Deusto. Senior Lecturer in the Department of Sociology and Social Work of the Universidad del País Vasco UPV/EHU. Her research focusses on communication technologies, everyday life, children and adolescents, and gender issues. Since 2006 she has been part of the EU Kids Online research network.

(iD https://orcid.org/0000-0002-8057-6370

Estefanía Jiménez Iglesias is a $\mathrm{PhD}$ in Audiovisual Communication from the Universidad del País Vasco UPV/EHU. Senior Lecturer in the Audiovisual Communication and Advertising Department of the UPV/EHU. Her research work focusses on the digital life of children and adolescents. She has been part of the EU Kids Online network since 2012.

(iD https://orcid.org/0000-0003-2897-5929

Nekane Larrañaga Aizpuru is a $\mathrm{PhD}$ in Sociology and Political Sciences from the Universidad de Deusto. Senior Lecturer in the Department of Sociology and Social Work of the Universidad del País Vasco UPV/EHU. Her research activity focusses on diversity, intercultural and interethnic relationships, education and values, bilingualism, identity, citizenship and acculturation.

iD https://orcid.org/0000-0002-8062-3544 
revista española de pedagogía año 77, n 273, mayo-agosto 2019

Spanish Journal of Pedagogy

year 77, n. 273, May-August 2019

\section{Table of Contents}

Sumario

\section{Educational and civic-penal responses to antisocial behavior}

Respuestas educativas y cívicopenales a los comportamientos antisociales

Guest editor: Fernando Gil Cantero

Editor invitado: Fernando Gil Cantero

\section{Fernando Gil Cantero}

Introduction: Educational and civic.penal responses to antisocial behavior

Presentación: Respuestas educativas y cívico-penales a los comportamientos antisociales

David Reyero, Fernando Gil Cantero

Education that limits is education that frees

La educación que limita es la que libera

Maria José Bernuz Beneitez, Esther Fernández Molina The pedagogy of juvenile justice: a child.friendly justice

La pedagogía de la justicia de menores: sobre una justicia adaptada a los menores
Marina Martins, Carolina Carvalho

What do teenagers lie about?

¿En qué mienten los adolescentes?

245
Inmaculada Méndez, Cecilia Ruiz Esteban, Juan Pedro Martinez, Fuensanta Cerezo

Cyberbullying according to sociodemographic and academic characteristics among university students Ciberacoso según características sociodemográficas y académicas en estudiantes universitarios

Irene Montiel, José R. Agustina

Educational challenges of emerging risks in cyberspace: foundations of an appropriate strategy for preventing online child victimization

Retos educativos ante los riesgos emergentes en el ciberespacio: claves para una adecuada prevención de la cibervictimización en menores

Maialen Garmendia Larrañaga, Estefanía Jiménez Iglesias, Nekane Larrañaga Aizpuru

Bullying and cyberbullying: victimisation, harassment, and harm. The need to intervene in the educational centre Bullying y ciberbullying: victimización, acoso y daño.

Necesidad de intervenir en el entorno escolar 


\section{Ana Rosser-Limiñana, Raquel Suriá-Martínez}

School adaptation and behavioural and emotional

problems in minors exposed to gender violence

Adaptación escolar y problemas comportamentales y

emocionales en menores expuestos a violencia de género

\section{Fanny T. Añaños-Bedriñana, Miguel Melendro}

\section{Estefanía, Rocio Raya Miranda}

Young women with protective and judicial measures

and their transit towards prison

Mujeres jóvenes con medidas de protección y judiciales y sus

tránsitos hacia la prisión

\section{Book reviews}

Esteban Bara, F. Ética del profesorado [Ethics of

teaching staff] (Juan García Gutiérrez). García

Amilburu, M., Bernal, A., \& González Martín, M.

313

R. Antropología de la educación. La especie educable [Anthropology of education. The educatable species] (Yaiza Sánchez Pérez). Rose, D., \& Martin, J. R. Leer para aprender. Lectura y escritura en las áreas del currículo [Learning to write/reading to learn: Genre knowledge and pedagogy in the SydneySchool: scaffolding democracy in Literacy Classrooms] (Francisco Lorenzo Bergillos). Buxarrais, M. R., \& Vilafranca, I. (Coords.). Una mirada femenina de la educación moral [A feminine view of moral education] (Eric Ortega González).

351

This is the English version of the research articles and book reviews published originally in the Spanish printed version of issue 273 of the revista española de pedagogía. The full Spanish version of this issue can also be found on the journal's website http://revistadepedagogia.org.

ISSN: 0034-9461 (Print), 2174-0909 (Online)

https://revistadepedagogia.org/

Depósito legal: M. 6.020 - 1958

INDUSTRIA GRÁFICA ANZOS, S.L. Fuenlabrada - Madrid 